\title{
NEURO-PHYSIOLOGICAL MEASUREMENTS FOR DIAGNOSIS USING EVOKED RESPONSES
}

\author{
K Siddique-e- Rabbani \\ Department of Biomedical Physics \& Technology \\ University of Dhaka, Dhaka 1000, Bangladesh \\ email: rabbani@univdhaka.edu
}

\section{Contents:}

1. Basic overview of the nervous system

1.1. The nervous system, digital communication, dendrites and axons

1.2. Source of action potential in neural tissue, propagation of action potential. Speeding up: Myelinated axons.

2. Basics of neurological measurements.

2.1. Measurement of propagating nerve action potential from a distance.

2.2. Electro Myogram (EMG)

2.3. Voluntary and Evoked responses.

2.4. Electro Encephalogram (EEG).

2.5. Signal averaging for evoked responses with noisy background.

3. Overview of Evoked responses \& clinical applications

3.1. Audio Evoked Responses

3.1.1. Slow Vertex Response (SVR)

3.1.2. Brain Stem Electrical Response (BSER)

3.1.3. Oto Acoustic Emission (OAE)

3.1.4. Evoked Responses from the Cochlea, Electro Cochleogram (ECoG)

3.1.5. Post Auricular Myogenic response (PAM)

3.2. Visual Evoked Responses

3.2.1. Visual Evoked Potential (VEP) from the brain

3.2.2. Evoked responses from Retina, Electro Retinogram (ERG)

3.3. Evoked responses from peripheral nerves, Sensory Nerve Conduction Velocity (NCV)

3.4. Evoked responses from muscles (Evoked EMG), Motor NCV

4. Neurophysiological measurement, basic ideas

4.1. Electrodes

4.1.1. Contact Potential and elimination

4.1.2. Polarisation and elimination

4.1.3. Motion artifacts and elimination: Floating electrodes

4.1.4. Electrical Equivalent circuit of an electrode

4.2. Equipment

4.2.1. Nerve Stimulator

4.2.2. Photo Stimulator

4.2.3. Audio Stimulator

4.2.4. Bioelectrical amplifier, necessity of differential measurement

4.2.4.1. Common Mode Rejection Ratio

4.2.4.2. Capacitor coupling of electrode

4.2.4.3. Patient safety and isolation

4.2.4.4. Gain and frequency requirements

4.2.4.5. Computerised data acquisition, analysis and display

5. Future and Discussion: Distribution of F-Latency (DFL) - a new physiological parameter with potential for improved diagnosis, conceived of and developed by the author's group

6. Bibliography 


\section{BASIC OVERVIEW}

\subsection{The nervous system, digital communication, dendrites and axons}

Suppose I get a pin prick unknowingly on the back of my hand, I shall instantaneously move my hand away. This reflex action is the work of my neuro-muscular system comprising of the peripheral nerves, different sensory endings, the connected muscles, and obviously the central nervous system comprising of the brain and the spinal cord as shown in Figure 1. The spinal cord may be assumed as an extension of the brain since certain simple decisions, as the reflex action mentioned above, takes place within the spinal cord. This also reflects an optimisation of our nervous system. If the command to take my hands away had to come from the brain, it could have been too late to avoid serious injury. However, information is simultaneously sent to the brain that such a thing happened.

The nervous system of the body performs all the responses and controls of the body that need very fast, almost instantaneous actions, within a fraction of a second. In contrast, endocrine system releasing hormones and other chemicals control and regulate the comparatively slower

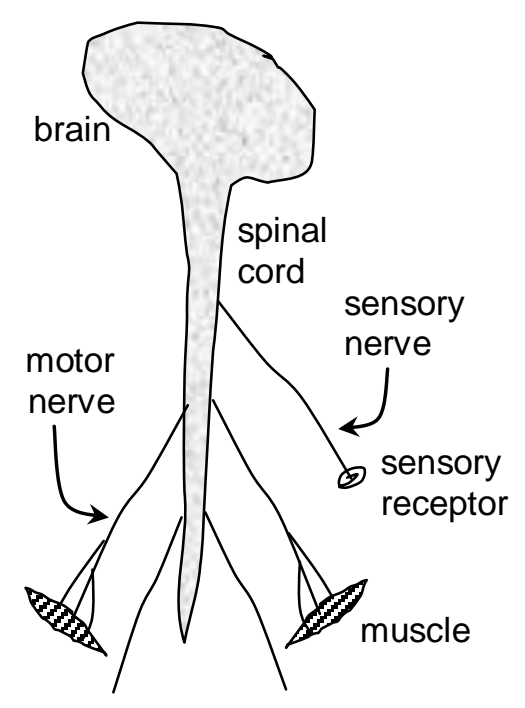

Fig.1: Overview of Nervous system functions that may take seconds to years.

The neuro-muscular system may be divided into two major categories - autonomous and voluntary. The autonomous system performs the basic acts of living, like the beating of the heart, pumping of the veins, movements of the stomach and intestine, secretion of chemicals from glands, etc., while the voluntary system is under our willful control, controlling actions like the movement of eyes, hands and legs as we see, talk and move around.

Physicists unfamiliar with the workings of these systems may get a surprise to know that the signals that move around in the nervous system are electrical, and more surprisingly, these are digital in character. Though the world around us is mostly analogue, we now know that for communication, digital techniques give much error free transmission; and in processing power, it surpasses that of analogue systems overwhelmingly. That is the reason digital communication and digital computers have revolutionised the world in the recent times, but the animal world 'knew' this and organised their bodies accordingly millions of years ago!

The peripheral nerves or 'nerve trunks' that we feel running through our hands and feet (same as the whitish cords of a few mm diameter that we see in animals cut open) each consists of thousands of nerve fibres or 'axons' varying in diameter from about a $\mu \mathrm{m}$ to about $20 \mu \mathrm{m}$. Some axons in a nerve trunk carry digital electric signals (called action potentials) from sensory endings such as the touch, pain and temperature sensing organs under the skin, the retina of eyes, and cochlea in the ear, the sensory organs in the nose and on the tongue, and from other organs of the body. These are the 'sensory' or 'afferent' nerve fibres which affect the decision making of the central nervous system. In response to the sensory stimuli, the brain or the spinal cord sends out action potentials through another set of axons called 'motor' or 'efferent' nerves which cause some motion to take place, as that of pulling the hand away in the above example. This gives us the differentiation between sensory and motor nerve fibres. These are essentially the same, but are different in the way they are terminated, and the direction in which they carry action potentials in nature. However, if we intervene unnaturally, that is if we stimulate both types of nerve fibres at any intermediate point artificially, action potentials will be propagated in both directions from the point of stimulation. It is just like an 
electrical wire, its use, or the direction of current through this wire depends on the designer of the circuit.

Coming back to the digital nature of nerve impulses, these are like the 1's and 0's in a digital electronic circuit; there is either a pulse of certain fixed height or none. The pulses are essentially of the same height and width, it is the frequency which varies with the intensity of stimulus as shown in Figures 2 and 3, the latter showing a logarithmic relationship. The brain knows precisely which sensory nerve is coming from which part of the body and what sense it is carrying, whether it is a feeling of touch from a specific point of a finger, or whether it is a particular frequency of sound coming from a particular ear, or whether it is one of the three primary colours coming from a specific point of the retina, and so on. Similarly when the brain or the spinal cord sends impulses to effect some action through a muscle, it also sends the required frequency of pulses through the specific nerve fibre, or a group of fibres. The muscles are also organised like the nerve fibres, there are hundreds and thousands of muscle fibres in a muscle that we are familiar with, and they also carry electrical impulses or action potentials. An axon usually divides into several branches before it connects to a muscle as shown in Figure 4, and each of these terminal branches connects to a muscle fibre through a neuro-muscular junction (from the end of the axon to the muscle fibre) within which the signal is transmitted through

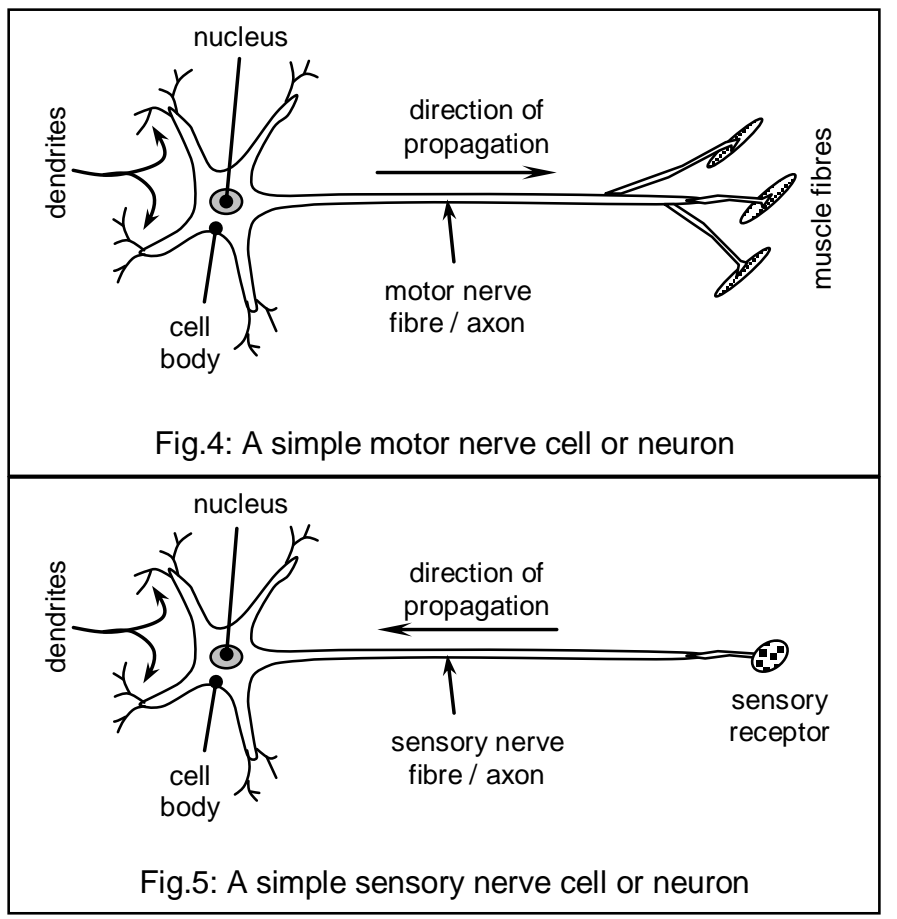
On receiving the chemical signal an electrical action potential is generated in the muscle fibre in turn, which is transmitted along its entire length, and a contraction of the muscle fibre takes place. Slightly different amounts of delay occur in each of the neuromuscular junctions due to which the contractions in the individual fibres within a group of muscle fibres served by a single axon (called a 'motor unit') spread out in time, with considerable overlap between them. Again a muscle that moves a certain part of a body usually contains many motor units served by many axons with slightly different conduction velocities causing further spread in time with overlap. Thus the resulting muscle contraction is smoothed out, an apparent analogue phenomenon, but obtained using digital techniques. 
Figure 4 also shows the other end of an axon, the cell body and dendrites, which may reside in the spinal cord, or in the brain. The total system comprising of the cell body, dendrites and the axon is a typical nerve cell or 'neuron'. This particular one shown is a motor neuron since it 'effects' or causes motion in a motor unit of a muscle. A simple sensory neuron as shown in Fig.5 will have a similar structure except the far end where it terminates on a particular type of sense receptor. Some peripheral sensory nerves may have a slightly different configuration with the cell body sitting not at the end, but somewhere in between. Various sensory receptors for touch, pain, heat, sound, light, sound, etc. exist from which information are sent to the central nervous system through respective nerve fibres. Within the spinal cord some of these sensory nerves make contact with cell bodies of motor neurons to cause a quick reflex action as mentioned in the beginning. There are sensory receptors even in muscles, in the form of structures in the middle called muscle spindles, which give information on the amount of contraction produced due to signals sent through motor nerves. This gives rise to a very important feedback mechanism for regulating the amount of muscle contraction. Dendrites are short lengths of conducting fibres meant for communication with other neurons over short distances within the brain or the spinal cord. These form the connections required for the very important functioning of the brain. On the other hand axons are long, the longest being about a metre, with the cell body in the spinal cord and the terminal point at the extreme end of the toe.

When I am talking, I am contracting different muscles of my mouth to different degrees at different times voluntarily, i.e., through my wishful control. The origin of this thought process for muscle control occurs in the brain, which then sends signals down appropriate nerve fibres to the muscles of my mouth, so that the requisite amount of contraction takes place in the appropriate muscle. Again a feedback is taken by the brain from the muscle spindle through sensory axons to assess the exact degree of contraction produced, and to modify the controlling signals to motor nerves accordingly, in order to get the exact desired contraction - no more, no less.

\subsection{Nerve action potential and propagation}

Axons are the nerve tissues through which nerve action potentials propagate over large distances, and this discussion will be limited to the nature of such action potentials and their propagation.

\subsubsection{Source of action potential in neural tissue}

a) An axon in resting state

A nerve axon may be considered as a tube

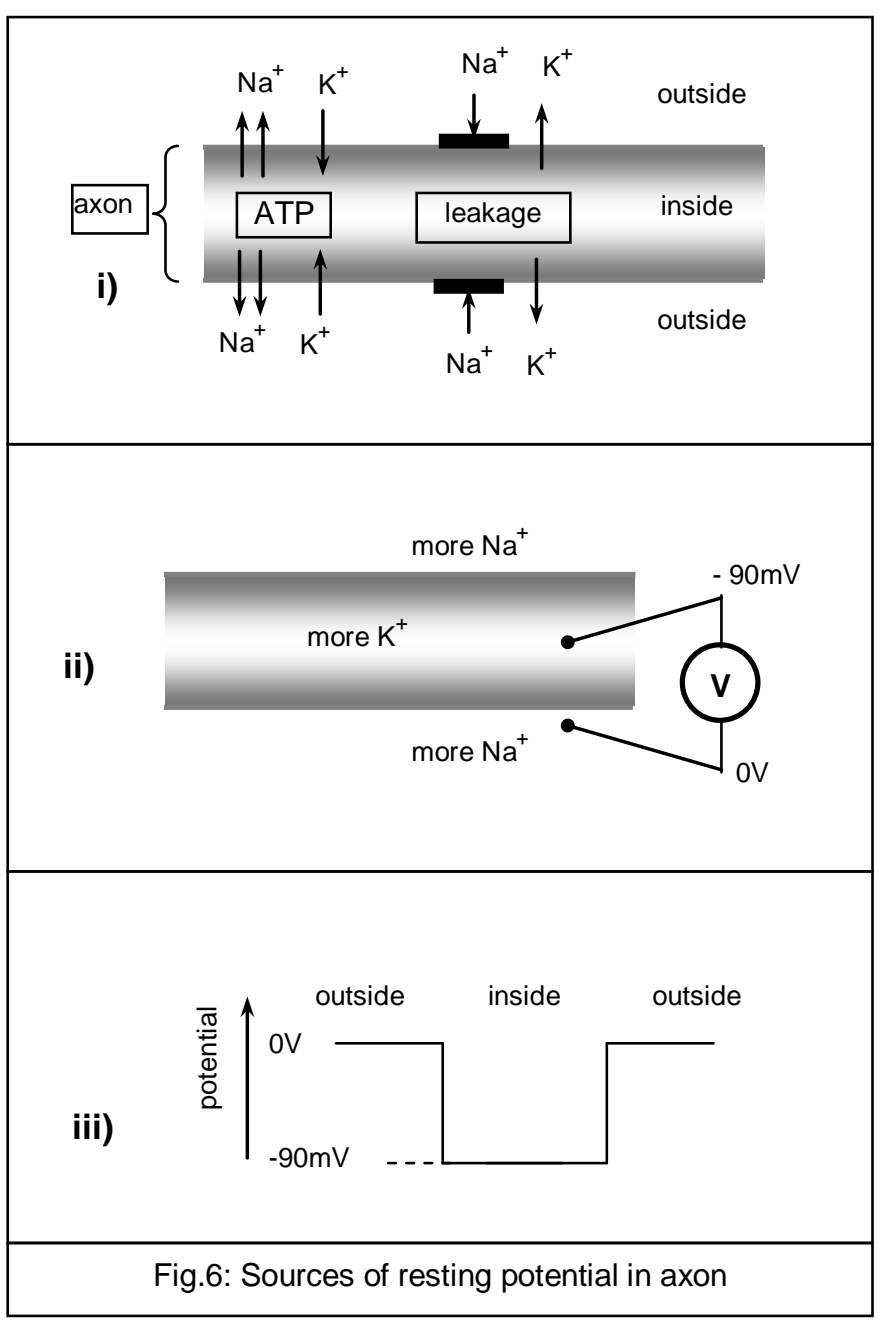
made of a thin electrically insulating membrane, with electrolyte inside. This is again embedded in body fluid, another electrolytic medium. The nerve membrane is semi-permeable to $\mathrm{Na}^{+}$ions in that it does not allow $\mathrm{Na}^{+}$ions to enter the axon from outside, but is permeable to $\mathrm{K}^{+}$ions which allows some $\mathrm{K}^{+}$ions to leak away to outside. A chemical called ATP (Adenosine Tri-Phosphate) continually forces $\mathrm{Na}^{+}$ions out from the inside as shown in Figure 6(i). This causes $\mathrm{K}^{+}$ions to move in from outside, but the rate of the latter process is 
slower than that of the former. This ends up in a high concentration of $\mathrm{Na}^{+}$and a low concentration of $\mathrm{K}^{+}$ions outside the axon, and a low concentration of $\mathrm{Na}^{+}$and a high concentration of $\mathrm{K}^{+}$ions inside [Figure 6(ii)].The ionic distribution ends up in such a state that about $-90 \mathrm{mV}$ is created just inside the membrane with respect to the outside as shown in Figure 6 (ii) \& (iii). This potential is known as the resting potential of an axon and the axon is said to be polarised as shown in a section of Figure 7.

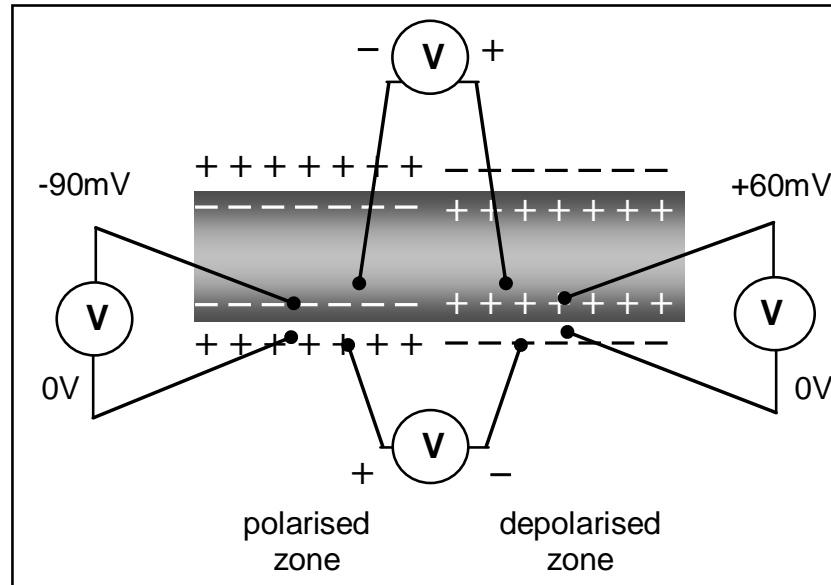

Fig.7: Polarised (resting) and depolarised zones and the potentials involved

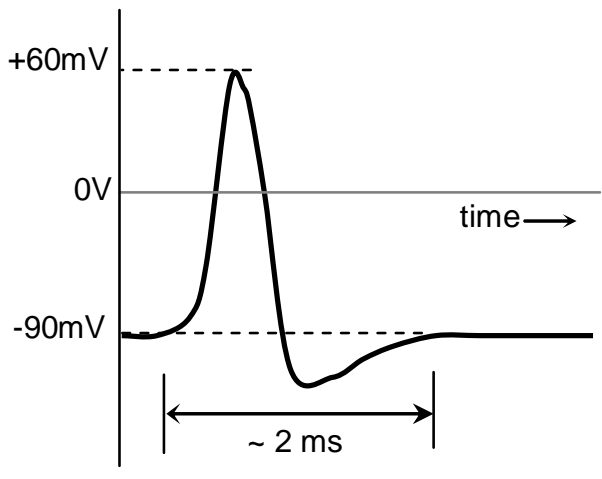

Fig.8: Nerve action potential (transmembrane potential at a point, varvina with time)

\section{b) Depolarisation of axon, action potential}

As mentioned before, normally the membrane does not allow $\mathrm{Na}^{+}$ions to enter. However, if by any means the transmembrane potential is reversed by about $20 \mathrm{mV}$ (i.e., the inside is at $-70 \mathrm{mV}$ with respect to outside), then the characteristics of the membrane changes, it becomes permeable to $\mathrm{Na}^{+}$ ions. As these ions enter the axon from outside, the transmembrane potential reverses further, and this increases the permeability of the membrane to $\mathrm{Na}^{+}$ions further causing a gush of $\mathrm{Na}^{+}$ions inside like an avalanche. In order to maintain a charge balance some $\mathrm{K}^{+}$ions cross over to the outside, but it is not enough. Within a fraction of a ms, a reversed potential of about $+60 \mathrm{mV}$ builds up across the membrane as shown in Figure 7. This state is known as depolarisation and the axon segment is said to be depolarised. After this period, the characteristics of the membrane suddenly changes back again, it does not allow any more $\mathrm{Na}^{+}$ions to get inside. To restore charge balance $\mathrm{K}^{+}$ions cross over to outside in large numbers and the potential gets back to the resting potential value (with a bit of overshoot) and the phase is known as repolarisation, which takes another fraction of a ms. However it is not quite the original situation as yet. Meanwhile ATP continues to 'pump' $\mathrm{Na}^{+}$ions out and $\mathrm{K}^{+}$ ions also travel back to inside gradually restoring the original depolarised state. A time diagram of the transmembrane potential at a single point of the axon is sketched in Figure 8. The whole process takes about $2 \mathrm{~ms}$, and this quick variation in transmembrane potential is known as the action potential of a nerve axon. It can be seen that the total amplitude of the nerve action potential is about $150 \mathrm{mV}(=$ $90 \mathrm{mV}+60 \mathrm{mV})$.

\subsubsection{Propagation of action potential}

The reversal of transmembrane potential in the depolarised region is associated with a localised reduction of positive charges outside the axon membrane, and a corresponding increment inside. As shown through the arrow paths in Figure 9(i), this results in a localised ionic current which flows from the adjacent polarised region $\mathrm{BC}$ to the depolarised region $\mathrm{AB}$ outside the axon membrane, and in a reversed direction inside the axon. This charges up the capacitor formed by the outside and inside fluids (as conductors) and the membrane (as the dielectric). The charging potentials in the polarised and the depolarised regions are shown by the extra +ve and -ve signs in the figure. It can be seen that this charging potential helps the repolarisation process in the depolarised region $\mathrm{AB}$, but in the adjacent resting or polarised segment $\mathrm{BC}$ of the axon, the transmembrane potential is reversed. When 
this potential reaches the above mentioned threshold of about $20 \mathrm{mV}$, the membrane becomes permeable to $\mathrm{Na}^{+}$ions and the sequence of events leading to depolarisation, as mentioned above, takes place in the region BC which eventually becomes fully depolarised as shown in Figure 9(ii). During this course of events, the region $\mathrm{AB}$ becomes repolarised.

In the next sequence of events current loops between regions $\mathrm{BC}$ and $\mathrm{CD}$ will cause the depolarised region to move to $\mathrm{CD}$ while $\mathrm{BC}$ will go to the repolarised state and $\mathrm{AB}$ to the resting polarised state as shown in Figure 9(iii). It needs to be mentioned that though the potential directions as shown by the + and - signs are the same in the polarised and repolarised regions in Figure 9(ii), the repolarised region has an immunity for a short period which prevents it from being depolarised again. Thus the depolarised region propagates in the direction from $\mathrm{A}$ to $\mathrm{D}$. This essentially explains how the action potential propagates.

As mentioned before, in nature, action potentials propagate from the end terminal towards the brain in sensory nerves as the action potential is originated at the far end by the respective sensory receptors. On the other hand in motor nerves the action potential originates in the brain or the spinal cord and propagates towards the extremity. The point to make is that there is a single natural direction of propagation of action potentials in any axon. However, it is possible to originate a depolarisation at any intermediate point of the axon artificially (through mechanical, electrical or chemical means). In that case the action potential travels in both directions from the point of stimulation. This is the situation that takes place when a nerve is artificially stimulated for measurement of conduction properties of nerves for diagnosis, or for physiological study to be described shortly.

\subsubsection{Speeding up: Myelinated axons}

It should be noted that the speed of propagation of nerve action potential, or the nerve conduction velocity (NCV) is almost directly proportional to the diameter for the same type of axon. The NCV due to the mechanism of propagation described above would be a few $\mathrm{m} / \mathrm{s}$ for typical axons which is not adequate for fast body controls remembering that some axons may be about a metre long. Therefore a special arrangement of intermittent myelin sheath around an axon has been designed by 'nature' to speed up the propagation by about ten times as shown in Figure 10. In between the

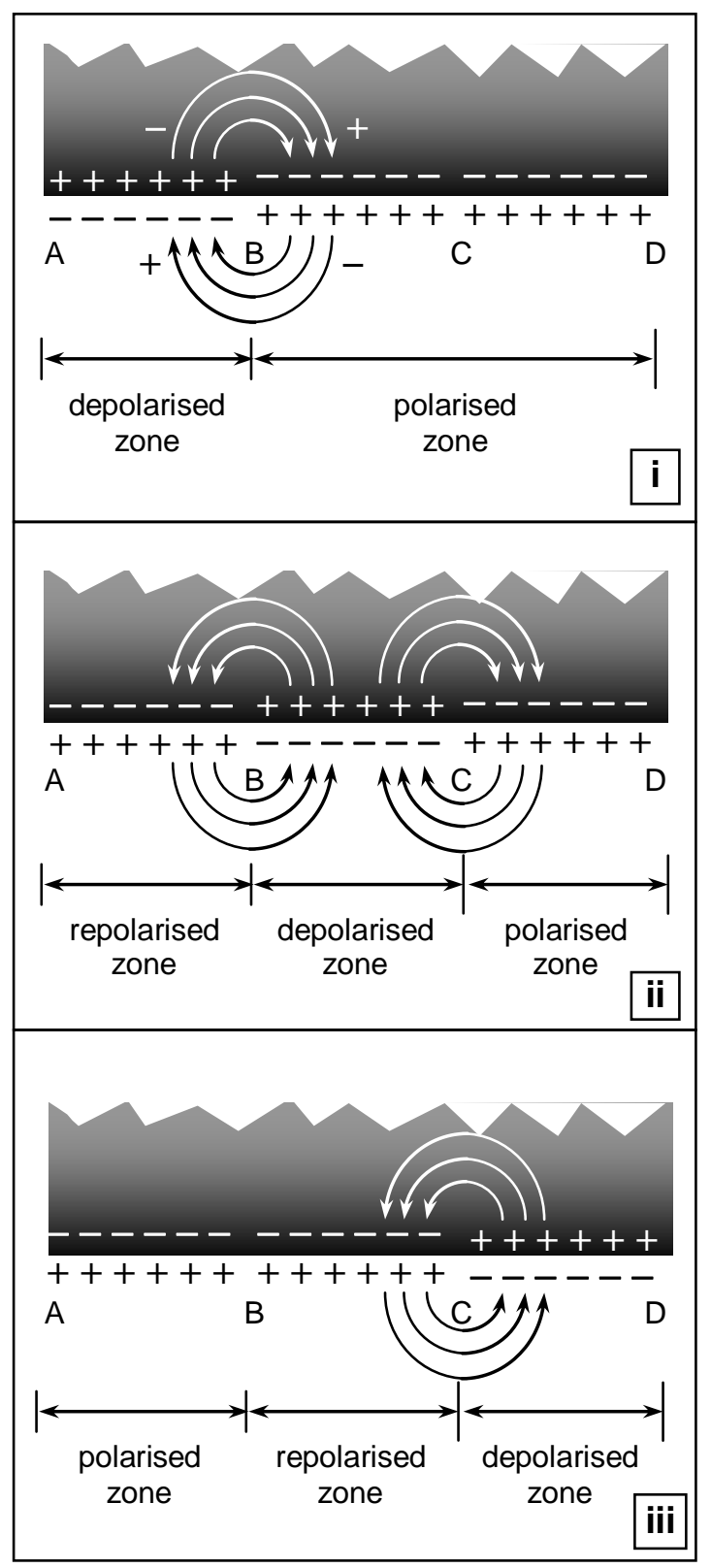

Fig.9: Propagation of nerve action potential through inter-zonal current loops

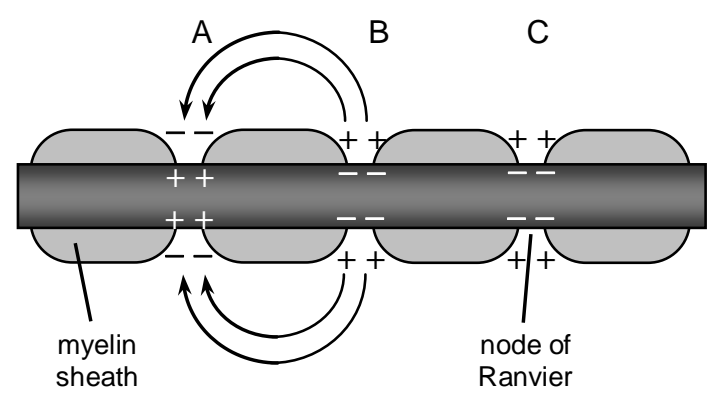

Fig.10: Myelin sheath and nodes of Ranvier speed up propagation through saltatory conduction 
myelinated segments (which are 1 to $3 \mathrm{~mm}$ long each) the axon is exposed to the body fluids directly at points called 'nodes of Ranvier'. The myelin sheath is a thick insulator which essentially blocks ionic charge transfer between the inside and outside of the axon, and the only places where ion transfer can take place are at these nodes. Suppose node A is depolarised while node B and C are at resting state. Now ionic loop current will take place directly between A and B skipping the intermediate zone under the myelin sheath. This will cause region B to be depolarised next without the necessity of depolarising the region adjacent to A under the myelin sheath. Therefore the depolarised zone jumps from node to node causing almost a tenfold increase in CV. This is known as 'Saltatory conduction'. The largest myelinated axons are about 15 to $20 \mu \mathrm{m}$ in diameter and have CV's almost reaching $100 \mathrm{~m} / \mathrm{s}$. Thus the signal needs about $10 \mathrm{~ms}$ to traverse a length of 1 metre through the fastest axons.

\section{BASICS OF NEUROLOGICAL MEASUREMENTS}

\subsection{Measurement of propagating nerve action potential from a distance}

In order to study or investigate neurological health measurement of NCV of nerves are necessary. Normally the measurements are taken using skin surface electrodes, away from the axons. With
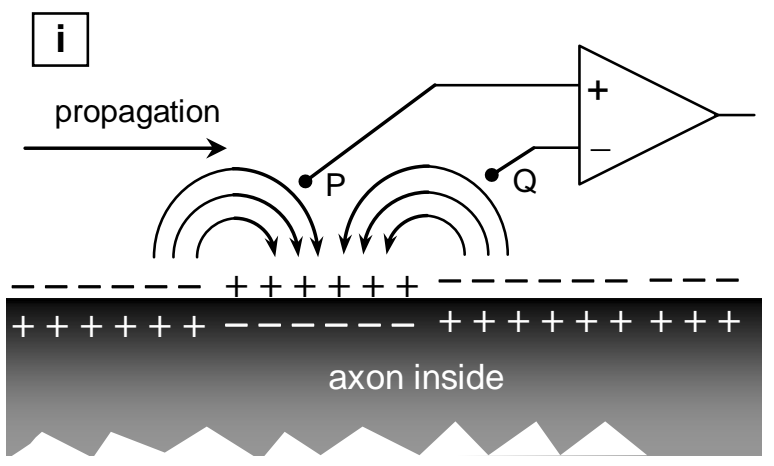

ii

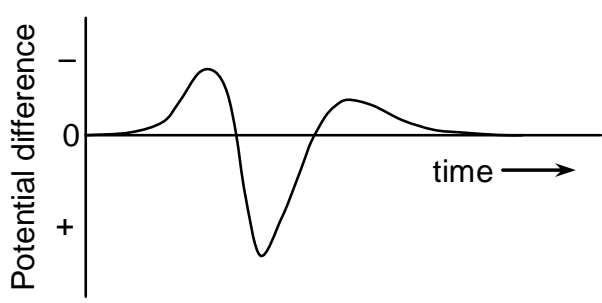

Fig.11: Potential picked up by electrodes depend on external circulating current pattern propagating along the axon (i), The resulting wave shape (ii) . respect to Figure 11(i), we can see that due to the external circulating currents in the body fluids between a depolarised region, and its adjacent polarised and repolarised regions in an axon, potential differences will be created between any two external points in the neighbourhood. As the depolarised zone moves along the axon, the potential between any two points (shown by $\mathrm{P}$ and Q) will change with time in a typical pattern as shown in Figure 11(ii). This has three peaks on both sides of zero (called triphasic) which can be explained based on the circulating current directions and their movement with time. In an actual measurement such waveforms from many axons (hundreds or thousands in a single nerve trunk) will combine to give a slightly different wave shape and amplitude. The amplitude will also be much reduced as one goes further away from the axon surface. Typical nerve action potentials measured using skin surface electrodes are only a few tens of $\mu \mathrm{V}$ in amplitude for evoked responses (to be described in the next section) while the internal nerve action potentials of individual fibres are much larger, about $150 \mathrm{mV}$ as mentioned before.

\subsection{Electro Myogram (EMG)}

As in nerve axons, a similar mechanism of action potentials also takes place in muscle fibres when action potentials from motor nerve fibres reach the neuro-muscular junctions called 'end plates', located almost at the mid-point of individual muscle fibres. Through a chemical neurotransmitter in these junctions a depolarisation is initiated in the muscle fibre and an action potential propagates in both directions causing a contraction of the fibre. The summing effects of a Motor unit and the whole muscle group have been discussed before. As for nerves, combined electrical action potentials of muscles can be picked up using electrodes on the skin surface which are called EMG or Electro Myogram (Greek 'myos' means muscle). Needle electrodes with very small exposed tips are also used to get localised EMG from a few muscle fibres. 


\subsection{Voluntary and evoked responses}

As mentioned before we can contract some muscles voluntarily, through the control of our own thought process. In such a case, different muscle fibres in the target muscle group contracts at slightly different times producing a combined pattern which is rather complex to analyse and is called the voluntary response, or voluntary EMG. Usually expert doctors perform the measurements using needle electrodes to obtain EMG from a few muscle fibres and to identify some patterns which have been related to certain disorders of muscles and nerves. On the other hand, we can stimulate a motor nerve trunk artificially at any suitable point using an electrical pulse of sufficient strength which produces action potentials simultaneously in all the nerve fibres in the nerve trunk. Propagating to the muscle group served by this nerve trunk it gives rise to a distinctive reproducible pattern of EMG. This is called an evoked EMG because the artificial pulsed stimulation evoked the production of this EMG signal. This signal is better understood and may be analysed to give some very positive inferences regarding certain neuro-muscular disorders. This tutorial lecture deals with evoked responses only not only from muscles, but directly from nerves and the brain as well.

\subsection{Electro Encephalogram (EEG)}

Electrical activity of the brain cells (neurons) also allow potentials to be measured using surface electrodes on the scalp. These are called EEG or Electro-encephalogram (Greek 'Enkephalos' stands for 'brain'). Since the brain contains more than 100 billion neurons, the normal EEG is very complex and difficult to analyse. People look for some gross pattern changes related to some known diseases or disorders. However, we can also pick up evoked EEG from the brain due to pulsed stimulation of specific nerves or sense organs which offer better understanding of some disorders and some of these will be discussed in this tutorial.

\subsection{Signal averaging for evoked responses with noisy background.}

Usually the signals from the brain, nerves and muscles are cluttered with unwanted noise of significantly large amplitudes. In the first attempt external noise signals which are almost the same all over the body called 'Common Mode noise', such as the 50 or $60 \mathrm{~Hz}$ mains line noise, are almost eliminated using special design of amplifiers and signal pick up systems described later in this tutorial. On the other hand, random unwanted signals may come from within the body itself in the case of evoked potential measurements. As an example when we want to measure the evoked potential from the brain due to a pulsed sound stimulus given to one of the ears, it is usually associated with normally occurring EEG from the billions of surrounding neurons as well, so much so that desired signal may be a few percent of the size of the unwanted EEG, which appears here as noise ( $\mathrm{S} / \mathrm{N}$ or signal to noise ratio has a fractional value in this case). We need the signal to be at least fifty to hundred times more than the noise to get a useful output. Fortunately for evoked responses, a technique known as signal averaging may be employed as described briefly below to achieve this goal.

In Figure 12 Graph (i) shows the stimulus pulse in time while Graph (ii) shows the resulting evoked potential that we want to pick up. Graph (iii) shows the evoked potential in association of a large random noise that is picked up in a single measurement. It can be seen that it is virtually impossible to find out any clue on the evoked potential, as the $\mathrm{S} / \mathrm{N}$ ratio is very small. Now if we apply the stimulus again we shall get a similar overall pattern, but the noise peaks will have different shapes and phases at different points in time since these are essentially random. Starting from the instant of the stimulus if we add up the two noisy evoked potentials some of the noise peaks appearing at opposite phases will cancel, and

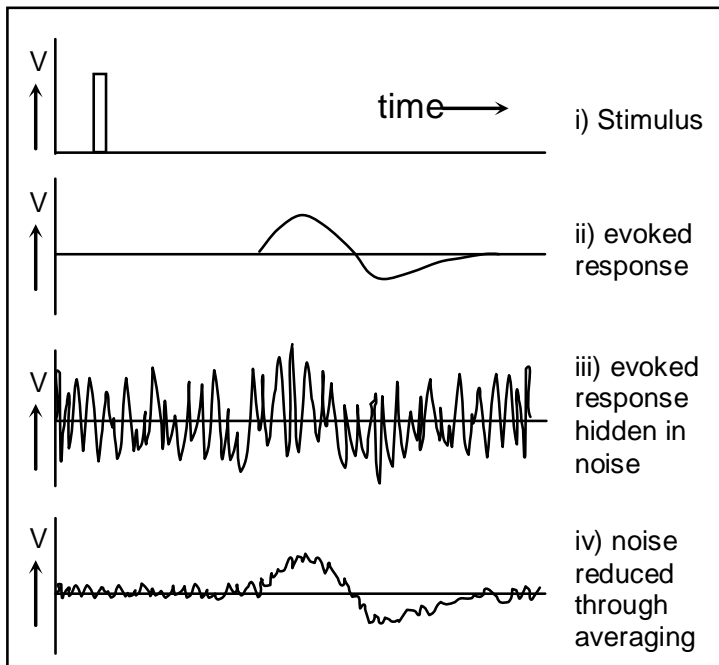

Fig.12: Illustration of signal averaging to extract evoked response hidden in large random noise 
those in phase will get enhanced, but the target evoked potential will be in phase, and will therefore get enhanced. Dividing the sum of the two potentials by 2 will keep the size of the evoked potential intact, essentially giving an average of the two evoked measurements. If we average many evoked potentials in this way, we would expect to get an overall reduction of the noise as the random noise peaks are expected to come at all points with different phases and would tend to cancel out while the evoked potential will add up and stand out as shown in Graph (iv). If we carry on doing this many more times eventually we will come to a point where the evoked potential stands out clearly. This is known as signal averaging and is used widely to get very small evoked potentials in a noise free condition. If ' $n$ ' is the number of times the averaging is performed (called the number of 'sweeps') then the $\mathrm{S} / \mathrm{N}$ improvement is given by 'square root of $\mathrm{n}$ '.

\section{OVERVIEW OF EVOKED RESPONSES \& CLINICAL APPLICATIONS}

\subsection{Audio Evoked Responses}

As the name implies these responses are obtained due to sound pulse stimulus given to one or both the ears. As will be detailed later, an amplifier used for such measurements requires three electrodes on the body, two are located across the source of the potentials, so that their difference gives the desired signal. One of these is sometimes called an 'active' electrode from whose potential the potential of the other 'reference' electrode is subtracted. These two form two inputs of a 'differential amplifier' while

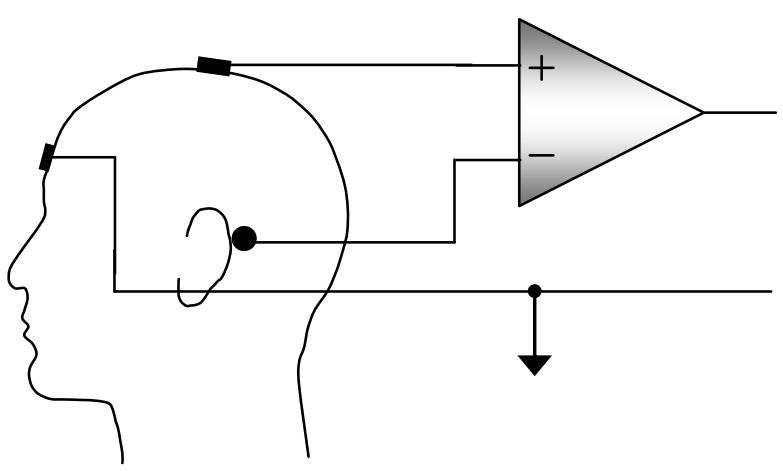

Fig.13: Electrode connection for SVR and BSER

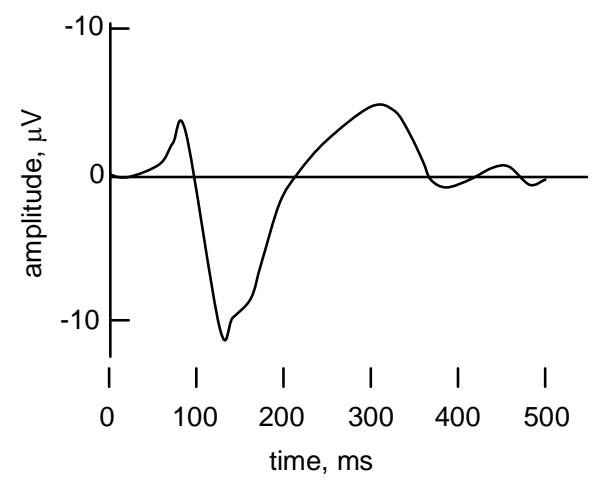

Fig.14: Typical Slow Vertex Response

a third electrode placed at a suitable location on the body provides a 'zero potential' point, or the 'common' electrode for both the above.

\subsubsection{Slow Vertex Response (SVR)}

This potential in response to a sound pulse stimulus to one of the ears is thought to be generated in the cortex of the brain. The active electrode is placed at the vertex of the brain as shown in Figure 13, while the reference electrode is placed over the mastoid bone behind the ear (the raised bony part), and the common electrode is attached on the forehead. The inputs to the amplifier are also shown. The stimulus is usually a tone burst of $300 \mathrm{~ms}$, a rise time of $10 \mathrm{~ms}$, and of a frequency of $1 \mathrm{kHz}$, applied though headphones or through a loudspeaker. The evoked response has a typical shape as shown in Figure 14 spanning between about 50 to $500 \mathrm{~ms}$ with an amplitude of about $10 \mu \mathrm{V}$. This is smaller than normal background EEG signal and about 30 responses, obtained once every two seconds, are averaged to obtain an acceptable evoked response.

SVR is usually employed to give an objective assessment of hearing loss, as opposed the normal audiometry where a response is asked from the subject. Such subjective responses may not be available properly from children, mental patients or from persons having an interest to obtain compensations from an opposing party for damage to hearing. The stimulus for SVR is sequentially reduced in strength to ascertain the threshold of hearing by observing the presence or absence of the evoked response. The frequency of the tone may be varied to obtain a frequency response of hearing. 


\subsubsection{Brain Stem Electrical Response (BSER)}

This response uses the same electrode placement as for SVR described above, but the amplitude is much smaller, less than about $0.5 \mu \mathrm{V}$ and occurring within about $10 \mathrm{~ms}$ of the stimulus as shown in Figure 15. This response is thought to originate from the Brain Stem. A tone burst of $5 \mathrm{~ms}$ duration and of frequency $2 \mathrm{kHz}$ repeating 20 times each second may act as the necessary stimulus. More than 2000 stimuli may be needed to obtain a reasonable noise free response. A click stimulus may also be used if frequency response of hearing is not desired.

\subsubsection{Oto Acoustic Emission (OAE)}

It is a relatively recent discovery (since late seventies) and still under development. The cochlea of the inner ear sends back a faint sound wave in response to a sound stimulus which can be picked up using a small microphone placed within the outer ear canal. This response, called the Oto Accoustic Emission (OAE) is absent in persons with hearing defects. This is particularly useful in infants and babies whose OAE is relatively large if normal. If any hearing deficiency is detected early in life remedial measures or fitting of hearing aids may help the baby to normal development. This measurement needs several thousand stimulations at a repetitive frequency between 50 and $100 \mathrm{~Hz}$.

\subsubsection{Evoked Responses from the Cochlea, Electro Cochleogram (ECoG)}

In this method the active electrode is a needle electrode which is inserted through the ear drum into a position close to the cochlea. The whole procedure is performed with the patient anaesthetised and is only done if it can be justified to study cochlear function in a patient. The reference and common electrodes are the same as for SVR. A small microphone within the outer ear canal produces tone burst stimulus as used for BSER and produce evoked responses that span over a few ms with amplitudes of several $\mu \mathrm{V}$. The stimulus are applied about 100 times a second and more than 200 stimuli are usually averaged.

\subsubsection{Post Auricular Myogenic response (PAM)}

In response to a sound stimulus our brain sends signals to contract the Post Auricular (PA) muscles (Greek: myos) behind the ears. In many animals this gives a 'startle' response (a twitch of the ears) to a sudden sound burst. It is not observed in human beings but an action potential of several tens of $\mu \mathrm{v}$ amplitude may be measured from the PA muscles occurring between about $10 \mathrm{~ms}$ and $50 \mathrm{~ms}$ after the stimulus. This response is observed from both sides even if the stimulus is given to a single ear only as shown in Figure 16.

The active recording electrode is placed at the base of the mastoid bone while the reference electrode is placed at the upper part of the mastoid bone. The common electrode is on the forehead as before. The sound stimulus is the same as for BSER. A few hundred stimuli are needed at a repetition

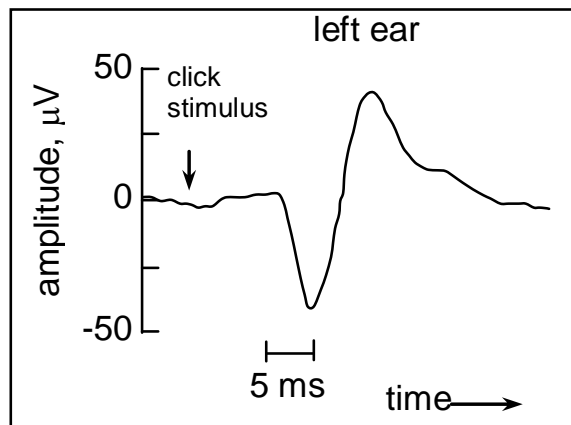

Fig.16: Typical Post Auricular Myogenic response from both ears due to click sound stimulus given to right ear only.

frequency of about $25 \mathrm{~Hz}$.

PAM is not a very reliable test as for about $20 \%$ of normal subjects this response is not available. However, its presence indicates a good hearing and is useful in screening children. 


\subsection{Visual Evoked Responses}

The retina of the human eye contains some 3 million cones and 60 million rods whose signals converge to about 400,000 fibres in the Optic Nerve. This section describes the following two evoked responses.

\subsubsection{Visual Evoked Potential (VEP) from the brain}

The part of the brain that performs the operations of vision lie at the back of our head (visual cortex). Two optic nerves carry the electrical action potentials generated at the retina of the two eyes to two sides of the visual cortex. Evoked responses may be obtained by stimulating one eye or both the eyes using pulsed checker patterns or simply pulses of light and recording from the visual cortex area at the back of the head. The active electrode is placed on the back of the head on the visual cortex area. The reference electrode is placed on the mastoid bone while the common electrode is placed on the forehead as before. The responses are a few $\mu \mathrm{V}$ in amplitude and hence needs few hundred repetitive sweeps at a frequency of about $1 \mathrm{~Hz}$.

VEP helps diagnose a disorder called Multiple Sclerosis (MS) in which degeneration of myelin sheath of nerve fibres in the central nervous system occurs. The latency to the peaks of VEP gets prolonged in MS because of conduction velocity reduction through the loss of myelin. VEP's obtained separately for the two eyes provide an indirect test for brain tumours which press onto one of the optic nerves but not the other. The compressed nerve will have a prolonged latency.

\subsubsection{Evoked responses from Retina, Electro Retinogram (ERG)}

Electrical action potentials from the retina due to a light flash can be obtained by placing the active electrode directly under the lower eyelid. A special carbon impregnated thread is used for this electrode. Alternatively a conducting but transparent electrode in the form of a contact lens may be placed directly over the cornea. The other electrodes may be placed as for the above evoked potential studies. The response is fairly large, of the order of $0.5 \mathrm{mV}$ and occurs within about $500 \mathrm{~ms}$ from the light stimulus. It may be obtained without signal averaging. The first peak of the response is absent in patients with inflammation of the retina that results in pigment changes, a disease called 'retinitis pigmentosa'.

\subsection{Evoked responses from peripheral nerves, Sensory nerve conduction velocity (NCV)}

Peripheral nerves are the nerve trunks coming out of the spinal cord and spreading out to all the peripheries of the body. The nerves in the upper and lower limbs (arms and legs) are subjected to such measurements frequently. Peripheral nerves like Median, Ulnar and Radial nerves in the arms and hands and Common Peroneal nerve, Tibial nerve and Sural nerve in the legs are commonly subjected to such measurements. The basic technique comprises of stimulation of the nerve trunk using a brief electrical pulse at one point, and recording of the resulting propagating action potential at another point in order to obtain a figure for the nerve conduction velocity (NCV). Usually such measurements are carried out employing skin surface electrodes and therefore the nerve trunk has to be available close to the skin at two points in the least. Techniques for electrical stimulation will be discussed in a bit of detail later.

A nerve trunk contains both motor and sensory fibres, therefore such measurements usually involve both types of nerves. However, by

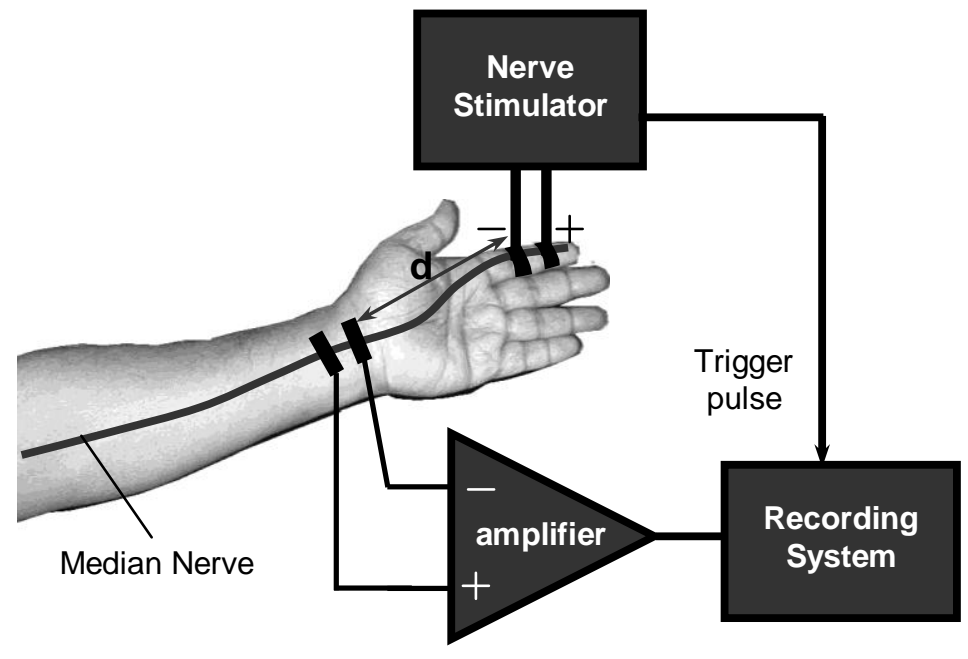

Fig.17: Technique for the measurement of sensory NCV 
stimulating at locations like fingers where there are no motor nerves, and recording at the wrist one may selectively measure Sensory NCV (SNCV). This may be done the other way round too, i.e., applying stimulation at the wrist and recording at the fingers. Figure 17 shows the technique for measurement on the Median nerve. The nerve is stimulated by applying a $0.1 \mathrm{~ms}$ wide and about $200 \mathrm{~V}$ high electrical pulse through two ring electrodes wrapped around the forefinger. The active and the reference recording electrodes are placed on the wrist, where the median nerve passes just below the skin. The evoked response is of the order of $30 \mu \mathrm{V}$ and occurs between about $2 \mathrm{~ms}$ and $5 \mathrm{~ms}$ of the stimulation. With a good system and good preparation of the skin it may be measured without signal averaging, but a few times of averaging

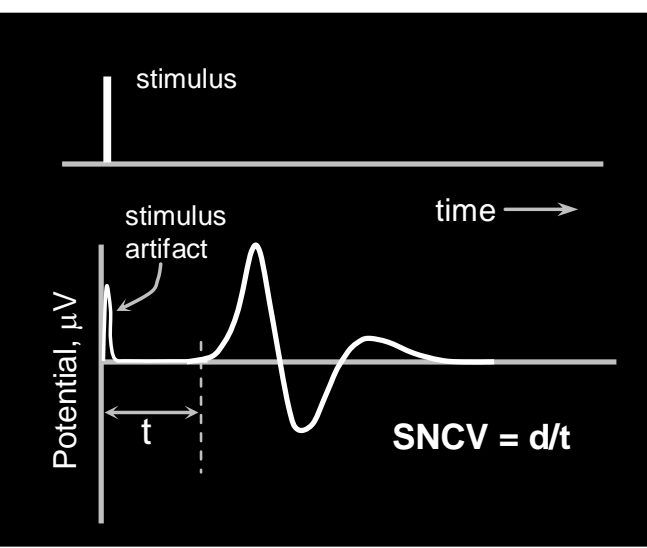

Fig. 18: Measurement of sensory NCV using evoked response from a nerve trunk. Stimulation pulse is also shown. gives a uncluttered signal. Figure 18 shows a sketch of an evoked potential similarly obtained. The stimulus is shown in the upper graph while the response obtained is shown in the lower diagram. The first peak appearing at the instant of the stimulating pulse is a stimulation artifact while the second wave shape is the desired response from the nerve. The latency $t$ (time delay of propagation) is usually measured to the onset of the resulting nerve response from the instant of stimulation. The distance $d$ between the points of stimulation and recording (Fig.17) are measured using a tape rule and SNCV is obtained as

$$
S N C V=d / t
$$

which is usually expressed in units of $\mathrm{m} / \mathrm{s}$. Typical normal values are about $45 \mathrm{~m} / \mathrm{s}$ or more for median nerve. A significant decrease in this value indicate some sort of disorder. A localised compression in the nerve pathway will reduce the NCV significantly, as will also be the case for degeneration of nerve fibres due to other causes. In the latter case the amplitudes will be reduced too. Therefore by looking at various parameters one can obtain a better diagnosis.

The nerve is stimulated under the cathode (will be explained later) which is therefore placed towards the wrist in the above experiment when stimulating at the finger. Again, the first peak of the response as recorded on the nerve pathway is usually negative. Traditionally clinicians want to see the first peak upwards in the display, therefore the first recording electrode on the path is connected to the inverting input (marked -) of the amplifier as shown in Figure 17 while the further electrode is connected to the non-inverting input (marked + ). The relevant distance $d$ of the nerve segment is measured between the cathode of the stimulating electrodes and the first (nearest) electrode of the recording pair as shown.

\subsection{Evoked responses from muscles (Evoked EMG), Motor NCV}

As mentioned before, when a motor nerve trunk is stimulated it gives rise to muscle action potentials in the connected muscle fibres which then propagates through the muscle fibres. The muscle fibres are more in number and are nearer to the skin surface, therefore the signals measured using skin surface electrode are much bigger than that obtained from the nerve trunks as mentioned above. As for nerve fibres, it is also possible to obtain an evoked muscle response (or evoked EMG) by stimulating the appropriate nerve trunk artificially. Typical amplitudes of evoked EMG's are between 5 and $15 \mathrm{mV}$ and are the largest biological signals measured. It is possible to measure NCV using evoked EMG as well. However, since there are unknown delays at the neuromuscular junctions it is not possible to get a figure for NCV using only one point of stimulation as was done for SNCV above. The unknown delay is eliminated by stimulating the same nerve trunk at two distant points and taking the difference of the latencies as shown in Figures 19 and 20. In this typical measurement set up for the median nerve, the recording electrodes are placed on the Thenar muscle which is the thick muscle at the base of the thumb. The active electrode is fixed at the belly of the muscle while the reference electrode is 


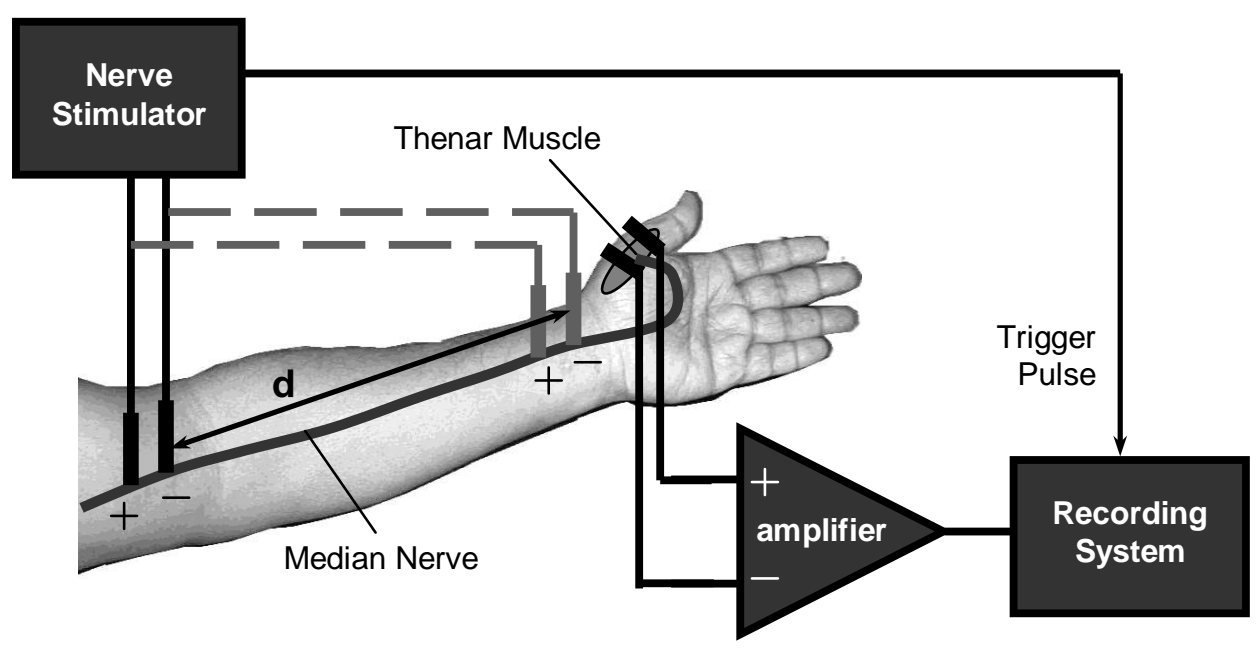

Fig.19: Technique for the measurement of motor NCV

placed a bit further away towards the thumb, on the tendon of the same muscle. The common electrode is placed either on the back of the palm, or at the wrist.

For nerve stimulation, at least two points are needed where the nerve trunk passes close to the skin. It is practically very difficult to stimulate nerves that lie deep under muscles and fat. Therefore, points where the nerve trunk lies close to the skin are chosen. For median nerve, such points are available near the wrist, elbow, arm-pit (axilla) and neck (Erb's point). Most of the initial measurements are made using wrist and elbow stimulation and this is shown in Figure 19. Stimulating at the wrist a response will be recorded on the muscle which is shown in the upper graph of Figure 20. The stimulation artifact is obtained as before which also helps in getting the starting time $(t=0)$. The time delay $t_{d}$ up to the onset of the response as shown is called the distal latency. When the nerve is stimulated at the elbow, the time delay $t_{p}$ is longer due to the longer path involved. This is called the proximal latency. The terms distal and proximal indicate spatial relative position with respect to the centre of the body. Between any two points, the nearer one is called proximal point, and the one further away is called the distal point. Now subtracting $t_{d}$ from $t_{p}$ will eliminate the unknown delay at the neuromuscular junction since it is included in both the latencies. The time delay $t_{p}-t_{d}$ essentially gives the time taken by the nerve action potential to travel through the nerve segment between the cathodes of the stimulating electrodes at wrist and at elbow. Measuring this distance $d$ ( Figure 19) using a measuring tape and dividing by $t_{p}-t_{d}$ will give the NCV of the motor nerves (MNCV) between the wrist and elbow as,

$$
M N C V=d /\left(t_{p}-t_{d}\right)
$$

If this is less than a standard threshold value, reduced conduction is suggested. However, the value varies greatly from person to person. Therefore, one needs to compare with NCV's obtained from different limbs of the same subject to say with confidence whether the $\mathrm{NCV}$ has decreased or not. By stimulating at various points of the nerve upto neck (Erb's point) as mentioned above, it is possible to identify localised entrapments or compressions, if there is any, and diffused neuropathies may be identified distinctively from localised ones. Such NCV tests may be carried out on most of the nerves in the upper and lower limbs and mentioned above in section 3.3.

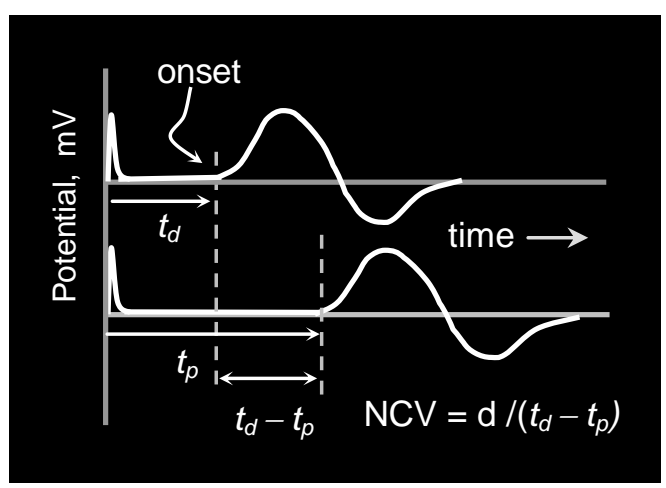

Fig. 20: Measurement of motor NCV using evoked EMG. Difference of latencies used to eliminate unknown delay at neuromuscular junction 
The distal latency only in the above study on the median nerve is also useful although unknown neuromuscular delay causes inaccuracies in such diagnosis. Approximate delays are known and significant increase in the latency may be related to entrapment or compression of the nerve in between the point of stimulation and the neuromuscular junction. The distal latency for the median nerve is usually about 3 to $4 \mathrm{~ms}$ for normal population. A very common disorder known as Carpal Tunnel Syndrome increases this latency significantly due to compression on the nerve just beyond the wrist, where it passes through a restricted zone called 'Carpal Tunnel'. If the distal latency is greater than $4.5 \mathrm{~ms}$, one may conclude that it is a case of CTS in most probability.

Similar studies can be made on Ulnar and Radial nerves in the hand and Common Peroneal and Tibial nerves in the legs.

There are nerves and muscles in the body where only one accessible point is available for stimulation rather than two on the same nerve. Here the latency may still be useful for a gross diagnosis. Comparison between the two sides of the same patient gives a better understanding of the abnormality in most cases, as two sides of the body are not similarly affected in most clinical conditions.

Peripheral nerve conduction studies provide definitive and objective tests and are widely used in neurological diagnosis.

\section{BASICS OF NEUROPHYSIOLOGICAL MEASUREMENT TECHNIQUES}

In this section measurement aspects and instrumentation of evoked responses from the peripheral nerves in the limbs (hands, legs) will be described. Any electrophysiological activity will create a current within the body as shown in Figure 21(i), since it is full of electrolytes, and therefore, is a volume conductor. These currents will cause potential drops between any two appropriate points, say P and Q, simply based on Ohm's law as shown in Figure 21(ii). Metal electrodes are needed to pick up these electrical potentials for subsequent amplification, processing and display in the measurement system

\section{1: Electrodes, problems and solutions}

Electrodes act as transducers in that these convert ionic current within the body to electronic currents in metallic wires outside. Metallic electrodes have certain characteristics which have to be understood in order to get useful measurement.

\subsubsection{Contact Potentials and elimination}

The human body can be considered as a bag of saline covered by a thin electrically insulating but leaky membrane, the skin. Therefore whenever a metallic electrode is brought into its contact several electrochemical processes take place. Just as in a battery cell, ionic exchange between a metal and an electrolyte causes potential barriers to form which in effect blocks further ionic transfer. This contributes to dc potentials but because of unstable reactions with underlying electrolytes, which again may be in motion, very low frequency $(<0.1 \mathrm{~Hz})$ components are also present. These low frequency components may be filtered out using high pass filters as most biological signals are much above such frequencies. Some metals give more stable potential than others and among the elemental ones, lead $(\mathrm{Pb})$ gives fairly stable potentials. However, most measurements use silver coated with silver chloride $(\mathrm{Ag}-\mathrm{AgCl})$ which has other benefits too as mentioned below.
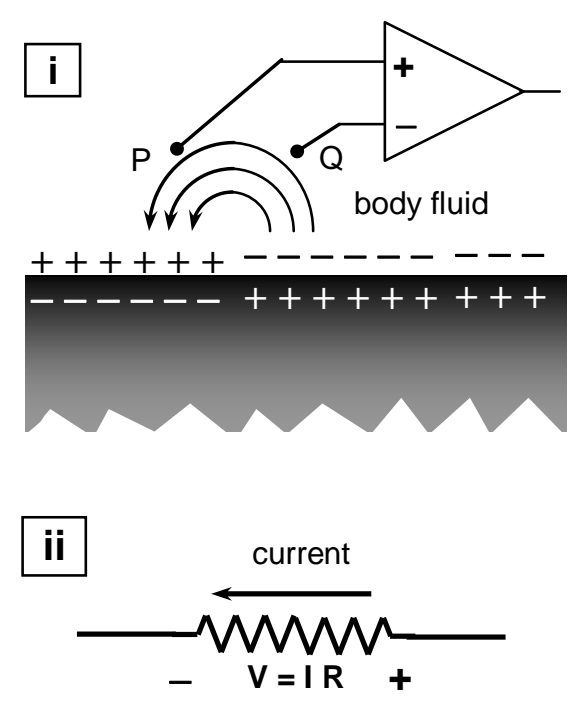

low pot.

high pot.

Fig.21: Mechanism of Potential difference between two electrodes placed around a nerve fibre 


\subsubsection{Polarisation and elimination}

If electrodes are dc coupled to amplifiers, a dc bias current may cause electrochemical reactions to form thin insulating layers of gas $\left(\mathrm{O}_{2}\right.$ and $\left.\mathrm{H}_{2}\right)$ underneath the electrodes, which causes build-up of charges on the two opposite sides of the gas layers. This is called polarisation and it contributes to a deterioration of the measured signals. One way to minimise this effect is to use an amplifier with very high input resistance and very low bias currents (needs FET's) so that the current drawn is very small. A better method is to use $\mathrm{Ag}-\mathrm{AgCl}$ electrodes where $\mathrm{Cl}$ acts as the charge carrier both in the electrolyte and in the

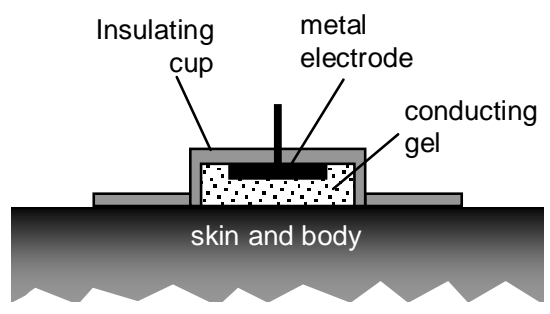

Fig.22: Floating electrode electrodes and causes electro-chemical reactions without the production of any gas. A third method would be the use of capacitor coupled inputs so as to block dc bias currents.

\subsubsection{Motion artifact and elimination: Floating electrodes}

The skin of any subject will move with occasional movement of the subject. This gives rise to fresh electrochemical reactions at newly exposed areas under the metal electrode, contributing to noise, called motion artifacts. To minimise this noise the metal electrode is kept afloat above a dense conducting gel which makes contact to the skin in turn as shown in Figure 22. A plastic cap like structure holds the arrangement in place whose outer flange is attached to the skin using adhesive gums. Straps or adhesive tapes may also be used to hold the electrode in place. The gel is also an electrolyte so that the electrochemical reaction is essentially shifted from the skin to the interface between the metal and the gel at the top of the plastic cup. The gel is highly viscous and has good surface adhesive properties so that any skin movement appears as a shear within the thick gel, no relative movement takes place between the metal electrode and the gel. This eliminates motion artifacts to a great extent.

\subsubsection{Electrical Equivalent circuit of an electrode}

The skin is a membrane which is essentially a leaky dielectric in electrical terms. It has a dead horny layer which provides a high resistance to electrical current. The arrangement of a metallic electrode on the skin may be modeled as Figure 23. Here the contact resistance appears in parallel with a capacitor and both appears in series with the contact potential. It is normally desired that the contact resistance be as

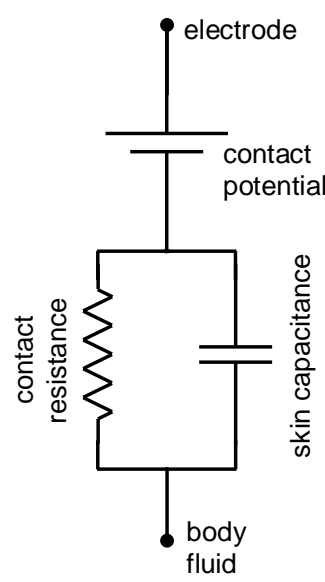

Fig.23: Equivalent circuit of a skin electrode low as possible, therefore the skin has to be cleaned well using spirit to remove grease and other dirt, and may need some abrasion to remove some dead tissue. The electrode needs a moist contact through the gel (water soaked pads work as well) which helps to reduce this

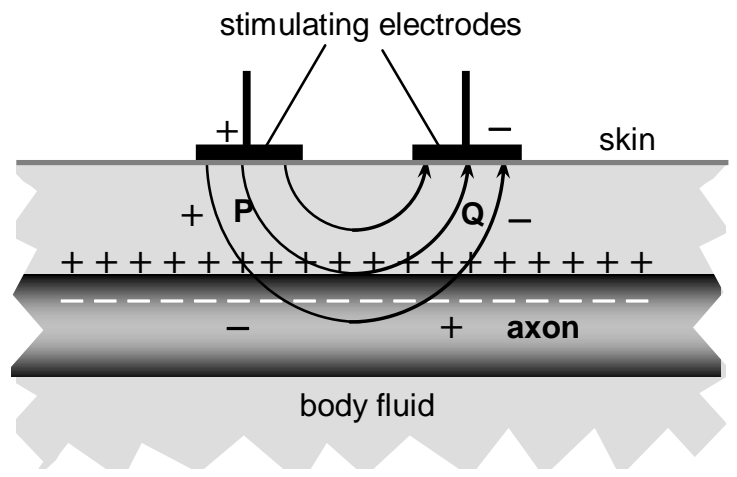

Fig.24: Mechanism of artificial electrical stimulation of nerve fibres (axon) contact resistance.

\subsection{Equipment for evoked potential measurement}

In this section equipment necessary for the evoked potentials will be described briefly, but firstly the mechanism of electrical nerve stimulation needs to be understood.

\subsubsection{Nerve Stimulator}

The basic mechanism of nerve stimulation is explained with respect to Figure 24. A current pulse is passed between two electrodes on the skin which are just above a nerve trunk. The current flows into the body tissue and the nerve and the 
situation for a nerve fibre, which is at resting potential (i.e., polarised), is shown here. The current crosses the membrane of the axon and enters inside at $\mathrm{P}$ and exits at $\mathrm{Q}$. Because of this external current the membrane potential at $\mathrm{P}$ is hyperpolarised in the same direction as before, so nothing new happens. On the other hand at $\mathrm{Q}$ the current causes a reversal of the membrane potential. As discussed before, when a reversal of about $20 \mathrm{mV}$ is affected, the axon membrane characteristics changes and the initiation of an action potential takes place. The action potential then propagates in both directions at the natural conduction velocity of the axon.

To create a depolarisation, a minimum of transmembrane current for a certain interval is needed. As can be visualised, most of the current flows in tissues just underneath skin, above the nerve fibre, and is wasted, and the deeper is the nerve, the more current is wasted. Therefore larger current is needed if the nerve is at greater depth. Typically for stimulating all the thousands of axons in a nerve trunk that passes just under the skin (cutaneous), a current pulse of about $20 \mathrm{~mA}$ to $50 \mathrm{~mA}$ over 0.1 to $1 \mathrm{~ms}$ period is needed. A smaller pulse width requires a larger current. To create this current the pulse voltage needs to be about $200 \mathrm{~V}$ to $300 \mathrm{~V}$. This explains why electrically stimulating a nerve deeper in tissue is very difficult. It will need so much current that it will not be tolerated by the subject.

A nerve stimulator can easily be made using the general purpose IC Timer 555 as shown in Figure 25. It is wired up to make a monostable multivibrator with control of pulse width through $R_{A}, R_{B}$ and $R_{C}$. The transistor is used to amplify the current pulse into the primary of a step up transformer. The secondary creates the necessary voltage and current pulse needed for nerve stimulation. The strength of stimulation may be controlled using a variable resistor in series as shown. A diode across the primary is needed to bypass induced high voltage in the transformer that might otherwise cause breakdown of the

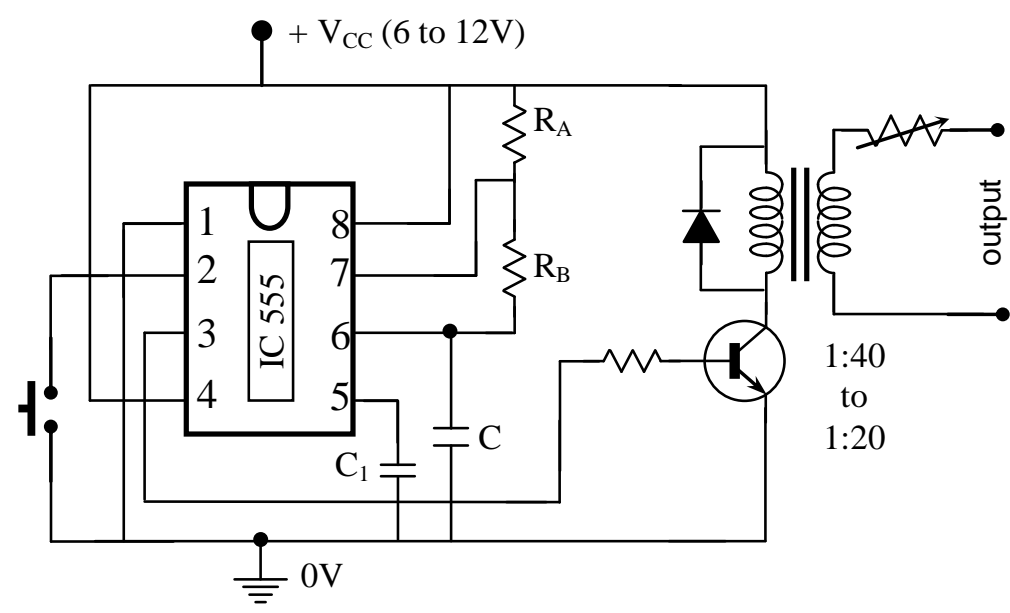

Fig.25: Basic nerve stimulator circuit made of a popular timer IC transistor. The stimulation pulse may be applied through any two metallic electrodes lined with some gel or water soaked cotton pads. A push button switch as shown may be used to trigger the stimulator. Alternatively, an astable multivibrator may be used to provide repetitive trigger at regular intervals as needed for signal averaging.

\subsubsection{Photo stimulator}

A xenon flash (as used for photo flash guns) may be used to provide light pulses of ms durations. It can be driven by an astable multivibrator. A white diffuser of about $200 \mathrm{sqcm}$ area need to be placed in front of the flash gun to provide an acceptable intensity from about a $50 \mathrm{~cm}$ distance. Alternatively, a CRT computer monitor may be used to produce these light flashes, but one needs to take care of the time needed for the raster display to build up. A multimedia projector may also be used to provide the stimulation with the screen placed at a distance. Most often a black and white checker board pattern (as a chess board) is switched between its normal pattern and its inverse (white for black and vice versa) to provide the stimulation. The size of the checker boxes are also varied to look for different aspects of eye function. 


\subsubsection{Audio Stimulator}

As mentioned briefly before, the stimulus may be click stimulus in which case a pulse output from an astable multivibrator may be used to drive a loudspeaker or a headphone after suitable amplification. If a tone burst is to be used one needs an oscillator of the desired frequency and a pulse generator as shown in Figure 26. Using an AND gate the tone burst may be generated to drive a loudspeaker or a

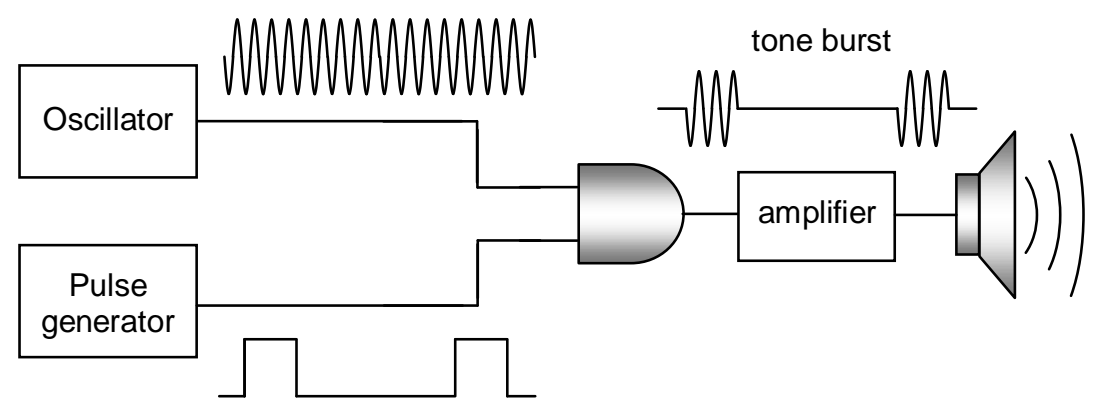

Fig.26: Tone burst generator headphone.

\subsubsection{Bioelectrical amplifier, necessity of differential measurement}

As can be understood from the above discussion that an amplifier has to amplify signals from a few

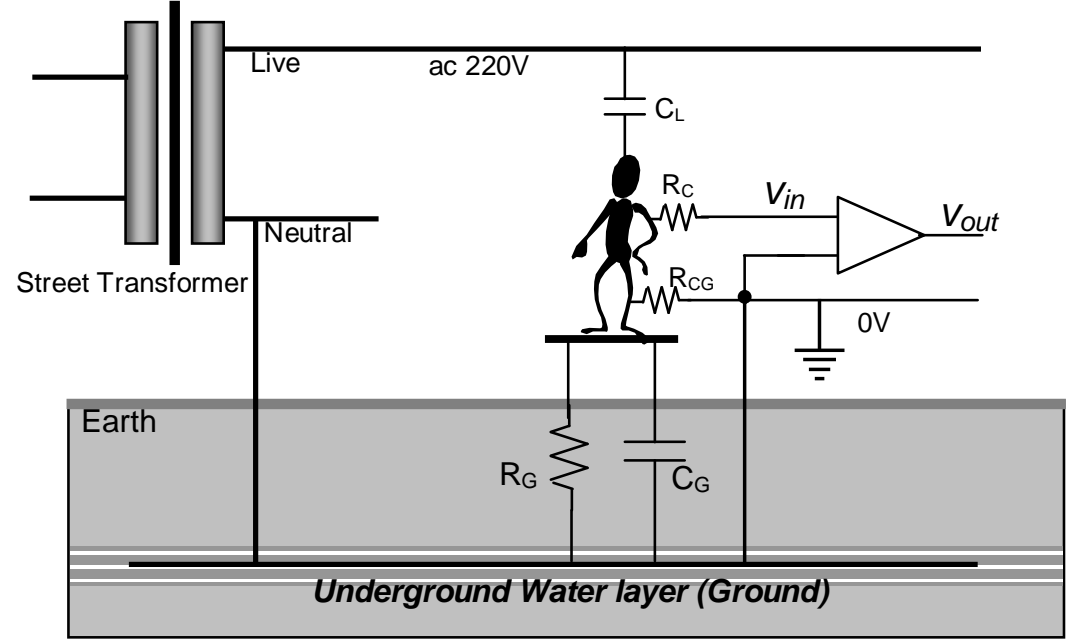

Fig.27: Source of $50 / 60 \mathrm{~Hz}$ noise $\mu \mathrm{V}$ to about $20 \mathrm{mV}$ for different evoked potentials. In typical situations in a building supplied by a mains power of $220 \mathrm{~V}$ or $110 \mathrm{~V}$ at 50 or $60 \mathrm{~Hz}$, our body picks up about $20 \mathrm{~V}$ with respect to ground because of a voltage divider action of stray capacitances $\left(\mathrm{C}_{\mathrm{L}} \& \mathrm{C}_{\mathrm{G}}\right)$ as shown in Figure 27. Here the ground means the water level below ground to which the neutral of the mains supply is connected. If we make a ground connection (using the third 'earth' or 'ground' pin of a

3-pin mains plug) to the body using an electrode, the skin resistance of about $10 \mathrm{k} \Omega\left(\mathrm{R}_{\mathrm{CG}}\right)$ helps reduce the above body voltage at 50 or $60 \mathrm{~Hz}$ to about $20 \mathrm{mV}$, a thousand fold reduction. This then appears as a noise to the desired signal, and as can be easily seen, is still far too high compared to the signal sizes that we want to measure. In the figure $\mathrm{R}_{\mathrm{G}}$ indicates leakage resistance to ground which is usually very high.

The mains line noise frequency of $50 / 60 \mathrm{~Hz}$ falls right in the middle of most of the bioelectrical signals that we want to measure, and therefore, cannot be filtered out without causing severe distortion of the signal itself, which is undesirable. Therefore alternative techniques have to be sought for.

Looking closely at Figure 27 together with the information that the body is like a bag of saline with very low resistance

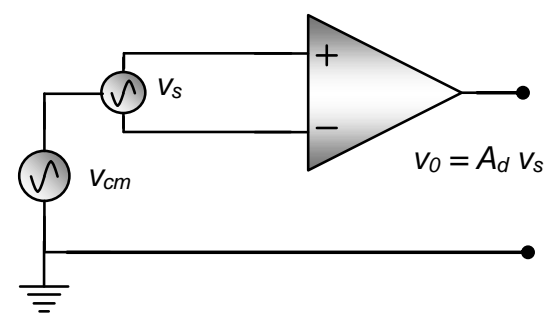

Fig.28: Differential amplifier with two inputs rejects common mode potential 
(typically a few hundred ohms), it may be appreciated that the potential picked up from the mains supply due to the voltage divider action of the capacitors and the ground electrode contact resistance is almost the same throughout the body (a few hundred ohms is negligible compared to the contact resistance of $10 \mathrm{k} \Omega$ to ground). This allows us to eliminate this noise through the use of a differential amplifier as shown in Figure 28 which accentuates the potential difference $\left(v_{s}\right)$ between the two input lines and rejects the potential $\left(v_{c m}\right)$ that appears the same (or, in common mode) in both of these with respect to the ground electrode, which is the mains line noise of $50 / 60 \mathrm{~Hz}$ in our case. We may model them as shown so that the potential at the non-inverting input $\left(v_{+}\right)$is $v_{c m}+v_{s} / 2$ while that at the inverting input $\left(v_{-}\right)$is $v_{c m}-v_{s} / 2$. The output of this difference amplifier is,

$$
\begin{gathered}
v_{o}=A_{d}\left(v_{+}-v_{-}\right) \\
=A_{d}\left[\left(v_{c m}+v_{s} / 2\right)-\left(v_{c m}-v_{s} / 2\right)=A_{d} v_{s}\right.
\end{gathered}
$$

where $A_{d}$ is the differential gain of the amplifier. This technique helps eliminate mains line noise to a great extent.

Therefore we can see that we need three electrodes to measure a bioelectrical potential (Figure 29), two ( $a$ and $b$ ) across the source of the potential, and the third (c) in any convenient position, to work as the 'common' or the 'ground'. We also note from the above discussion that the magnitude of the common mode noise depends very much on the proximity of mains line equipment and the contact resistance $\mathrm{R}_{\mathrm{CG}}$ of the common electrode. Therefore, it is better if the subject is moved away from mains connected equipment as much as possible, and the contact resistance of the ground electrode should be as small as possible. Good skin preparation, and a large area electrode are desirable for this electrode.

Figure 29 also shows contact resistances $\mathrm{R}_{\mathrm{C} 1}$ and $\mathrm{R}_{\mathrm{C} 2}$ at

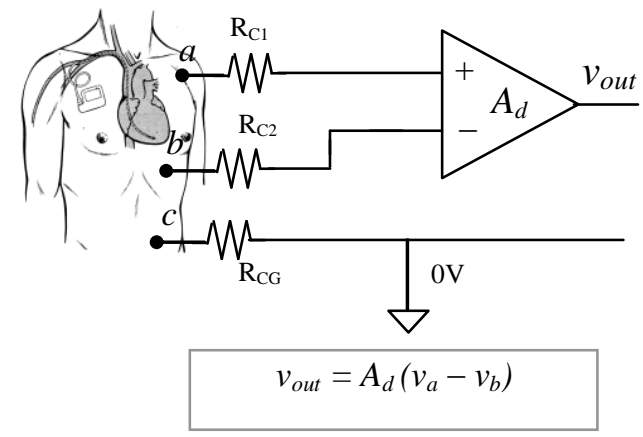

Fig.29: Differential input across source of bioelectric potential with respect to common electrode, attached at any other point on the body. Electrode contact resistances are also shown.

electrodes a and b respectively, which are also of the order of $10 \mathrm{k} \Omega$ but may differ widely between them. These contact resistances appear in series to the respective input terminals of the amplifier. Therefore the input resistances of the amplifier, for both the differential input terminals, should be at least 100 times greater than the highest contact resistance encountered. Assuming the highest contact resistance to be $20 \mathrm{k} \Omega$, the input resistances of both the input terminals of the differential amplifier should be greater than $2 \mathrm{M} \Omega$.

\subsubsection{Common Mode Rejection Ratio (CMRR)}

The above equation for the differential gain is ideal in that the output due to common mode input $v_{c m}$ is zero. In practice it is not so and it is a great challenge to remove this common mode noise noting that it is hundreds or thousands of times greater than the desired signal in some cases. A practical differential amplifier (usually called an Instrumentation amplifier) will have a output given by,

$$
v_{o}=A_{d}\left(v_{a}-v_{b}\right)+A_{c m} v_{c m}
$$

where $A_{c m}$ is the net amplification of the common mode input potential, or, the common mode gain. The ratio of the two gains is called the Common Mode Rejection Ratio (CMRR) as:

$$
C M R R=A_{d} / A_{c m}
$$

It is obvious that we would like CMRR to be as large as possible. Practical instrumentation amplifier integrated circuits (IC) are available which have CMRR's of 100 to $120 \mathrm{~dB}$, i.e., with corresponding ratios of $10^{5}$ to $10^{6}$, and these are adequate to get noise free signals down to about a few tens of $\mu \mathrm{V}$ (as for EEG from brain). For smaller evoked potentials signal averaging technique as mentioned before 
gives an excellent solution. It is possible to make an instrumentation amplifier (also may be called a bioelectrical amplifier) using operational amplifier IC's and a common circuit is presented in Figure 30. Here op-1 and op-2 has to be closely matched and therefore has to be taken from a dual or a quad opamp IC where these are made on a single chip and undergo the same processing steps. The CMRR will be degraded if these are not closely matched. The variable resistance shown is used to match the the resistors associated with this part of the circuit to obtain the maximum CMRR.

\subsubsection{Capacitor coupling of electrodes}

As mentioned before, to eliminate polarisation it is advisable to have capacitor coupled inputs, which essentially are high pass filters, and these are shown

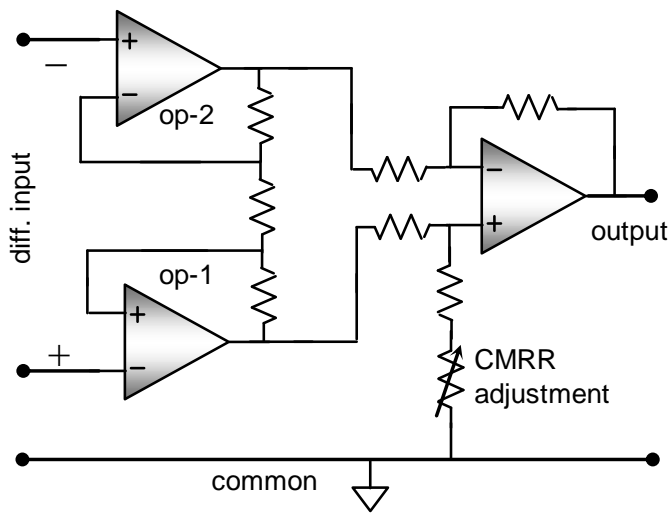

Fig.30: Instrumentation/ Bioelectric amplifier using op-amp IC's. in Figure 31 where the instrumentation amplifier is shown as a block. These coupling capacitors have another advantage. If any of the front end op-amps gets damaged and if the dc supply of the op-amps are connected directly to patient electrodes, a dc current may will flow and cause ulceration if the current is greater than about few tens of $\mu \mathrm{A}$. This may happen even if the supply voltage may be as low as $5 \mathrm{~V}$ only. The capacitors block such dc currents and provide patient safety. Again, these capacitors and resistors at the two differential inputs have to be as closely matched as possible to minimise the deterioration of CMRR.

One has to understand all the aspects of the introduction of mains borne noise into the measuring system in order to reduce the common mode noise effectively. It is clear from the above discussion that contact resistance of the ground or 'common' electrode should be as low as possible. Mismatch of electrode resistances will also cause a deterioration. Again the input leads and the ground or common wire form closed loops. Magnetic field created by mains driven transformers, motors, etc.

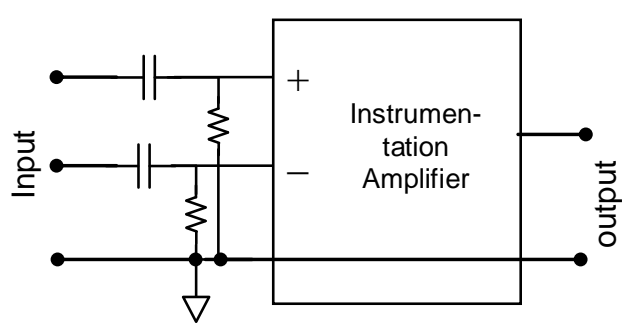

Fig.31: C-R high pass filter coupling at inputs. in the proximity will generate an induced emf in these loops, which if different at the two inputs, will add to the common mode noise voltage. Therefore the two input leads should be run as close as possible, minimising the area created by their looping.

\subsubsection{Patient safety and isolation}

Since most of these equipment have mains connected displays, graph recording equipment, etc., one

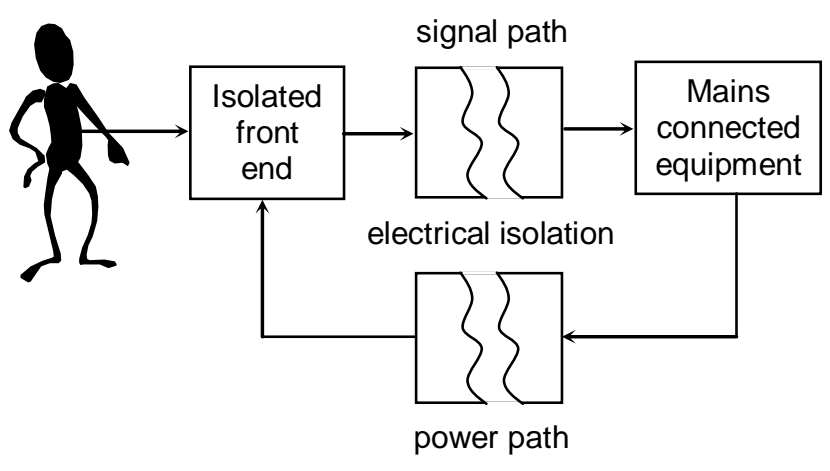

Fig.32: Electrical signal and power isolation for patient safety has to consider the safety of the patient from the hazards of electrical shock. The whole equipment has to be designed such that in case any of the circuitry gets damaged and the mains voltage gets to the low voltage circuit, the patient should remain safe. For this reason an electrical isolation is mandatory in all hospital equipment. The basic scheme is shown in Figure 32 . Both the low voltage dc power and signal paths to and from the patient connected front end have to be electrically isolated against such accidents. The leakage currents through these isolation circuitry 
cannot be greater than about $10 \mu \mathrm{A}$. This requirement comes from a possibility of microshock which may happen if heart patients having an exposed electrical connection direct to the heart are connected to such equipment. This may occur for patients who are being tested for the fitting of a pacemaker. A current greater than $50 \mu \mathrm{A}$ through these direct routes to the heart may be fatal. Normal transformers that are used for common low voltage equipment may have higher leakage currents (due to interwinding capacitance and coil to core leakage path) and are not adequate for protection needed in hospital equipment. Specially built low leakage transformers may be used for such isolation. Optical isolation (through the use of LED and photo-diode) is more popular for the signal section. Batteries may be used for the front-end power to provide good isolation, but one needs to consider the possibility of finding a flat battery in an emergency!

\subsubsection{Gain and frequency requirements}

How much total gain is needed depends on the signal size and the amplitude needed to drive subsequent graph plotting or display circuits. Many such instruments have a standard input of $\pm 1 \mathrm{~V}$, and this can give a guide to the overall gain needed. In the description of the different evoked potentials the signal sizes were discussed. For example for an evoked EMG of $10 \mathrm{mV}$ an overall gain of 100 will be needed, while for an evoked BSER of about $1 \mu \mathrm{V}$, the desired overall gain would be $10^{6}$.

The frequency response requirement will also depend on the frequency content of the particular signal. However, it has to be remembered that any filter, whether high pass or low pass, has a phase shift associated with it. At cut-off frequency this shift is about $45^{\circ}$ which is significant. The effect is illustrated with the help of Figure 33. Suppose a signal having two frequency components $f_{1}$ and $f_{2}$ is passed through a high pass filter with the cut-off at or near $f_{1}$. Now for the component $f_{2}$ the phase shift is almost zero, but $f_{l}$ will suffer a phase shift of about $45^{\circ}$. At the output the combination of these two components will not be the same as the input which we wanted to amplify. Thus there will be significant distortion in the signal which is undesirable. For this reason the cut-off frequencies for both high pass and low pass filters are removed several times beyond

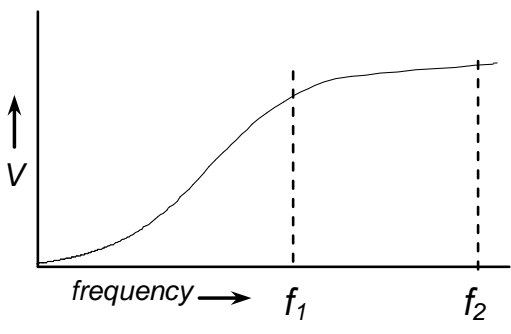

Fig.33: Origin of phase distortion the respective significant frequency components of the particular signal being amplified. For example, for evoked EMG, the significant frequency components may lie between about $10 \mathrm{~Hz}$ and a few $\mathrm{kHz}$. Therefore the lower cut-off of the high pass filter should be set not above 2 or $3 \mathrm{~Hz}$, while the upper cut-off of the low pass filter section should not be set below about $10 \mathrm{kHz}$.

\subsubsection{Computerised data acquisition, analysis and display}

Computer or microprocessor based data acquisition is almost universally employed nowadays for all evoked potentials measurements. This allows complex data analysis and display of measured data and software control on data acquisition. Signal averaging can also be performed very easily through modification of the software. Computerised data acquisition poses a challenge to any research worker, but if the basics are well understood, a person having an electronics and computer background can perform the work easily. A basic computerised data acquisition system is described in the appendix which will satisfy the quest of the interested ones.

\section{FUTURE AND DISCUSSION}

Evoked potentials have lot to offer in terms of diagnosis and physiological study, but we have extracted very little of the information hidden. There is a lot of scope for research in these areas. Somatosensory evoked potentials (SSEP) obtained from the brain through stimulation of any peripheral nerve gives information of the conduction around the central nervous system which is not possible with normal NCV measurement. 
Conventional NCV measured from evoked nerve and muscle action potentials are only obtained from the onset of the responses, which corresponds to the fastest nerve fibres only, it does not give any information of the health of the slower axons in a nerve trunk. In many disorders relatively slower nerve fibres are affected, not the fast ones. Patients show clinical symptoms of neural disorder, but the conventional NCV measured from the onset does not give any clue. It is like the timing obtained for reaching the finishing tape by the winner in a 100 metre sprint in athletics from which a velocity value may be obtained. No clue is obtained from this velocity, however, regarding the performance distribution of all others finishing later. What we want to know is the velocity distribution of all the runners, including the backtrackers - how many runners fall within a particular narrow velocity group, etc. We want exactly the same information of all nerve fibres in a nerve trunk in an ideal diagnosis, which is called the distribution of conduction velocity (DCV) but no satisfactory practical technique has been developed so far. The amplitude and the shape of the evoked response may have such information hidden and several groups throughout the world are trying to extract DCV from such responses, but again these have not turned out to be reliable. A simple experimental technique developed by the author and his group in Bangladesh recently may be a significant contribution to this end which appears to give DCV of some motor nerve fibres directly and appears to be sensitive enough to identify certain disorders at early stage.

As mentioned before, if a motor nerve is artificially stimulated at any point, action potentials are generated within the individual fibres which travel in both directions. The ones that travel towards the cell bodies in the spinal cord die out mostly, but a few percent of the cell bodies back-fire after a brief delay, and send fresh action potentials down the nerve fibres to the muscle. These produce a delayed compound muscle action potential called the F-response which has a small amplitude. The shape, amplitude and latency of F-responses vary on repeated trials; sometimes there will be none. This variation is known to happen because of randomness of the event; which nerve cells will backfire is not previously known.

Based on the above information the author conceived of the new method described below to obtain an approximate DCV of some type of motor nerve fibres in a nerve trunk. The above situation is similar to a box containing hundreds of coins of a few denominations (say, 5 or 6 ), completely mixed up. If we are now asked to approximately determine the number of coins of each denomination in a short time, not counting all the coins, we can do the following experiment which is based on statistical probability. Let us draw a single coin and note down its denomination. We now return the coin to the box and shake it vigorously to mix them well. We again draw a single coin, note down its denomination, and return it to the box and shake it again. In this way we repeat the same procedure for about 30 times. Now if we plot a graph with the coin denominations in the horizontal axis and the number of times that each denomination came out in our 30 draws, we will get a frequency distribution of coin denominations, and may say that the coins in the box has approximately the same distribution of occurrence, with certain statistical uncertainty. We would expect this result since each draw was a random event, the probability of a coin denomination to come up in each draw would be proportional to its actual number in the box. The author applied exactly this concept to this new technique for getting the DCV involving the F-responses.

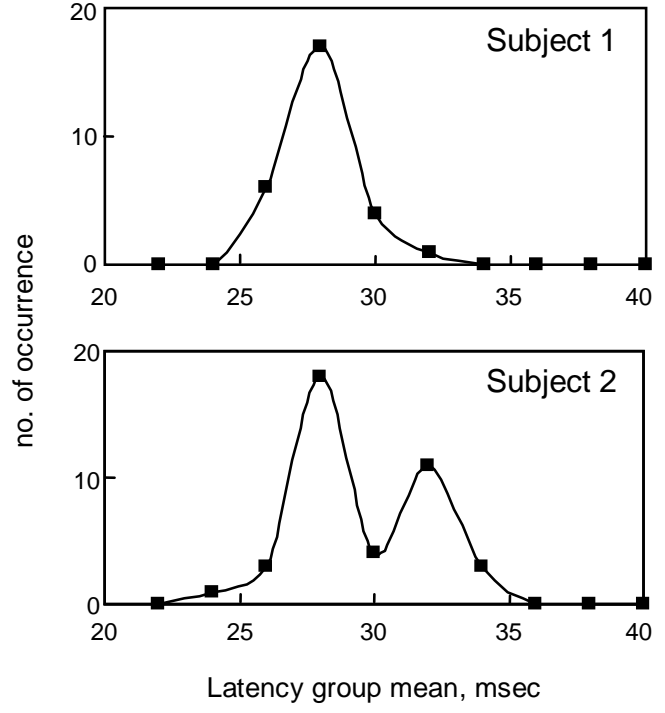

Fig.34: Distribution of F-Latency (DFL) from two subjects. Upper : normal. Lower: having Cervical Spondylosis 
About 30 F-responses were obtained from the same nerve trunk of a particular subject with adequate time delays between each stimulation (to allow restoration of resting situation) and their F-latencies (to the onset) were noted. These were then divided into $2 \mathrm{~ms}$ groups to give a new distribution which the author's group called distribution of F-latencies (DFL). Since latency is inversely proportional to velocity, DFL will be inversely related to DCV. To test whether this hypothesis of relating DFL to DCV is right they obtained DFL from the same subjects several times, and all of them correlated very well showing almost the same pattern each time. Of course there were some variations which were expected for a statistical experiment of such kind. The above agreement was found to be valid through studies on many subjects too. Figure 34 shows DFL's from the median nerves of two subjects. As mentioned before, for each subject the DFL's obtained at different times match very well. Therefore DFL has physiological significance and this is a new information, and is a significant discovery of the author's group. This reproducibility also verifies the claim by the author's group that DFL is a direct reflection of DCV. Actually a mirror image of DFL around the vertical axis would be a good approximation of a DCV since latency is inversely proportional to velocity. Thus this simple and direct technique has the promise of providing significantly improved physiological study and diagnosis.

The preliminary study has also revealed that DFL is indeed a sensitive parameter, it has the potential of diagnosing certain neural disorders at an early stage. In fact Fig.34 itself has such an indication. Subject 1 was normal and had no neurological complains while subject 2 had diagnosed Cervical Spondylosis (CS), a neurological disorder which affects most of the people above the age of 50. In CS either the spinal cord is compressed due to a bulging disc in between the cervical vertebra (Myelopathy), or a bony growth in the vertebra compresses onto nerve roots coming out of the spinal cord (Radiculopathy). Both result in neurological symptoms in the peripheral limbs served by the respective nerves. In Figure 34 DFL demonstrates a double peak for the subject with CS in contrast to the single peak obtained from the normal and healthy subject. These patterns have been found to be consistent in other normal persons and subjects having CS. Therefore double peak in DFL may be an important indicator for diagnosis of CS. In some subjects a broad peak was obtained and we think of this as an indicator for an early stage of CS, which is manifest even before the subject feels of any symptoms. We are working on the underlying physiological causes of this double peak and working on some hypotheses. Preliminary results appear to provide some support to the hypotheses. Currently investigative tests for CS are Contrast-CT and MRI both of which are expensive, not available widely, while the former is traumatic to the patient as well. If our new method using DFL bears out, it would be a much cheaper diagnostic technique, requiring virtually zero running cost except the initial cost of the equipment and the time of the clinician.

From the above work it appears that a Physicist has a lot to offer in neuro-physiological studies, particularly in electrophysiology. A close collaboration of medical Doctors and Physicists may reveal many more secrets of the neurological systems which will eventually lead to new and improved methods for the understanding of body functions, for diagnosis of diseases and disorders, and therapy.

Besides, very few of the Third World countries have facilities for such measurement since such equipment tend to be very expensive if imported, and the investigations are time consuming. However, the technology is within the capability of Electronic Engineers and Physicists in most Third World countries, and these can be designed and fabricated at a fraction of the cost of imported equipment. The research work done by us employed a computerised equipment we built locally with expertise gained through a British collaboration in the eighties.

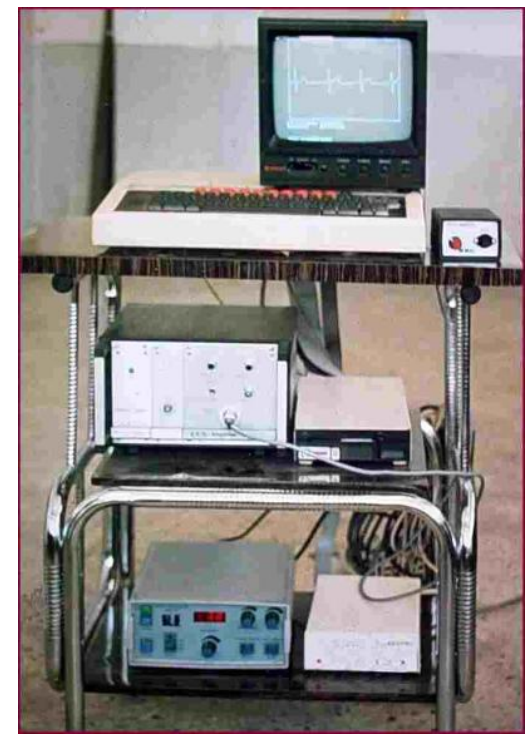

Fig.35: Computerised electrophysiology equipment made by the author's group locally in 1988 
A picture of the first prototype is shown in Fig.35 which used a microcomputer of that period. Later we switched to an IBM compatible microcomputer keeping the same front end amplifier. We designed the necessary analogue interface card and the necessary software afresh, completely indigenously. This was useful in that we could redesign any hardware or software to incorporate any new research idea, which was the case in the above work on DFL.

It is possible to design and fabricate most of the modern medical equipment in the Third World. What is needed is expertise mainly in three areas, namely, sensors, electronics and computers (both hardware and software) besides that in traditional engineering fields which are already available in all Third World countries. If such equipment are made locally, cost of procurement will come down sharply, and the useful life of the equipment will increase manifold since the expertise for repair and maintenance would be available locally, including supply of spares. Only then it will be possible to provide modern health care to the masses. At present hardly one percent of the people living in the Third World can get modern health care in real terms, and it is a failure on the part of us, the scientists. We cannot eliminate the economic disparity existing throughout the world overnight, but through innovation and courage we can create a bypass route, and can bring modern healthcare to the doorsteps of the common person in our countries. This will also allow us to contribute with new ideas and techniques, as the above example of DFL indicates.

\section{BIBLIOGRAPHY}

1. Medical Physics and Physiological Measurement, Brown and Smallwood, Blackwell Scientific Publications.

2. Medical Physics, Cameron and Skofronick, John Wiley \& Sons.

3. Medicine and Clinical Engineering, Jacobson and Webster, Prentice Hall Inc.

4. Kimura J. Electrodiagnosis in diseases of nerve and muscle: Principles and practice, 2nd Ed. Philadelphia, F.A. Davis company; 1989.

5. Rabbani KS, Alam MJ, Salam MA. Frequency Distribution of F-Latencies (DFL) has physiological significance and gives Distribution of Conduction Velocity (DCV) of motor nerve fibres with implications for diagnosis. J of Biol Phys. (Springer), 2007;33:291-303.

6. Alam MJ, Rabbani KS, Possible detection of cervical spondylotic neuropathy using Distribution of Flatency (DFL), a new neurophysiological parameter, BMC Research Notes, 3:112, 2010, <http://www.biomedcentral.com/1756-0500/3/112>.

7. Hossain MI, Chowdhury EA, Mamun AA, Salam A, Baig TN and Rabbani KS. Use of distribution of Flatency (DFL) in the detection of cervical spondylotic neuropathy, Bangladesh J of Med Phys 2011;4:3742.

8. Rabbani KS. Hypotheses to explain the occurrence of multiple peaks of DFL in nerve conduction measurement. Bangladesh J of Med Phys 2011;4:27-36. 
